\title{
ON FUSION RULES AND SOLVABILITY OF A FUSION CATEGORY
}

\author{
MELISA ESCAÑUELA GONZÁLEZ AND SONIA NATALE
}

\begin{abstract}
We address the question whether the condition on a fusion category being solvable or not is determined by its fusion rules. We prove that the answer is affirmative for some families of non-solvable examples arising from representations of semisimple Hopf algebras associated to exact factorizations of the symmetric and alternating groups. In the context of spherical fusion categories, we also consider the invariant provided by the $S$-matrix of the Drinfeld center and show that this invariant does determine the solvability of a fusion category provided it is group-theoretical.
\end{abstract}

\section{INTRODUCTION}

Throughout this paper we shall work over an algebraically closed field $k$ of characteristic zero. Let $G$ be a finite group. An important invariant of $G$ is given by its character table, defined as the collection

$$
\left\{\chi_{i}\left(g_{j}\right)\right\}_{0 \leq i, j \leq n},
$$

where $\epsilon=\chi_{0}, \ldots, \chi_{n}$ are the irreducible characters of $G$ over $k$ and $e=g_{0}, \ldots, g_{n}$, are representatives of the conjugacy classes of $G$. Several structural properties of $G$ can be read off from its character table. For instance, the character table of $G$ allows to determine the lattice of normal subgroups of $G$ and to decide if the group $G$ is nilpotent or solvable. See [15, pp. 23]. It is known, however, that the character table of a finite solvable group $G$ does not determine its derived length [18, [19].

In particular, if $G$ and $\Gamma$ are finite groups with the same character table, then $G$ is solvable if and only if $\Gamma$ is solvable. In addition, the knowledge of the character table of a finite group $G$ is equivalent to the knowledge of the structure constants, in the canonical basis consisting of isomorphism classes of irreducible representations, of the Grothendieck ring of the fusion category $\operatorname{Rep} G$ of finite dimensional representations of $G$ over $k$, so-called the fusion rules of $\operatorname{Rep} G$.

The notions of nilpotency and solvability of a group $G$ have been extended to general fusion categories in [14, [12. Let $\mathcal{C}$ be a fusion category over $k$. Then $\mathcal{C}$ is nilpotent if there exists a series of fusion subcategories

$$
\text { Vect }=\mathcal{C}_{0} \subseteq \mathcal{C}_{1} \subseteq \cdots \subseteq \mathcal{C}_{n}=\mathcal{C},
$$

and a series of finite groups $G_{1}, \ldots, G_{n}$, such that $\mathcal{C}_{i}$ is a $G_{i}$-extension of $\mathcal{C}_{i-1}$, for all $i=1, \ldots, n$. On the other side, $\mathcal{C}$ is solvable if there exist a sequence of fusion categories Vect $=\mathcal{C}_{0}, \ldots, \mathcal{C}_{n}=\mathcal{C}, n \geq 0$, and a sequence of cyclic groups of prime order $G_{1}, \ldots, G_{n}$, such that, for all $1 \leq i \leq n, \mathcal{C}_{i}$ is a $G_{i}$-equivariantization or a $G_{i}$-extension of $\mathcal{C}_{i-1}$. See Subsection 2.2. Some features related to nilpotency and solvability have been extended as well from the context of finite groups to

Date: December 17, 2015.

2010 Mathematics Subject Classification. 18D10; 16 T05.

Key words and phrases. fusion category; fusion rules; solvability; $S$-matrix.

This work was partially supported by CONICET and SeCYT-UNC. 
that of fusion categories; remarkably, an analogue of Burnside's $p^{a} q^{b}$-theorem was established for fusion categories in [12].

It is apparent from the definition of nilpotency of a fusion category $\mathcal{C}$ given in 14 that this property depens only upon the Grothendieck ring of $\mathcal{C}$, that is, it is determined by its fusion rules. In this paper we address the question whether the solvability of a fusion category $\mathcal{C}$ is also determined by its fusion rules.

Since a solvable fusion category has nontrivial invertible objects and a simple group has no nontrivial one-dimensional representation, then no solvable fusion category can have the same fusion rules as a simple finite group. We show that if $\mathcal{C}$ is a fusion category with the same fusion rules as a dihedral group, then $\mathcal{C}$ is solvable. On the other hand, if $\mathcal{C}$ has the fusion rules of a symmetric group $\mathbb{S}_{n}$, $n \geq 5$, then $\mathcal{C}$ is not solvable; Theorem 3.4 and Corollary 3.7

We study some families of examples of non-solvable fusion categories arising from representations of semisimple Hopf algebras associated to exact factorizations of the symmetric group $\mathbb{S}_{n}$ and the alternating group $\mathbb{A}_{n}$. For a wide class of such fusion categories $\mathcal{C}$, we show that $\mathcal{C}$ cannot have the fusion rules of any solvable fusion category. See Theorems 4.14, 4.18, 4.20 and 4.22.

In the context of braided fusion categories, the solvability of a fusion category $\mathcal{C}$ is related to the existence of Tannakian subcategories of $\mathcal{C}$; it is known that if $\mathcal{C}$ is a non-pointed integral solvable braided fusion category, then it must contain a nontrivial Tannakian subcategory [29, Lemma 5.1].

We show that if $\tilde{\mathcal{C}}$ is a non-pointed braided fusion category which has the same fusion rules as a solvable fusion category $\mathcal{C}$, then $\tilde{\mathcal{C}}$ contains a nontrivial Tannakian subcategory. See Theorem 5.1.

For a spherical fusion category $\mathcal{C}$ we study a somehow stronger invariant, analogous to the character table of a finite group, consisting of the $S$-matrix of the Drinfeld center $\mathcal{Z}(\mathcal{C})$ of $\mathcal{C}$. Indeed, the $S$-matrix of a modular category $\mathcal{D}$ is usually named the 'character table' of $\mathcal{D}$ in the literature; see for instance [13. A celebrated formula due to Verlinde, and valid for any modular category, implies that the $S$ matrix of $\mathcal{Z}(\mathcal{C})$ determines its fusion rules. We call two spherical fusion categories $S$-equivalent if their Drinfeld centers have 'the same' $S$-matrix; see Subsection 6.3.

We prove in Theorem6.5 that the $S$-matrix of the Drinfeld center does determine the solvability of a group-theoretical fusion category. That is, if $\mathcal{C}$ and $\mathcal{D}$ are $S$ equivalent spherical fusion categories and $\mathcal{C}$ is group-theoretical, then $\mathcal{C}$ is solvable if and only if $\mathcal{D}$ is solvable. We also show that being group-theoretical is a property invariant under $S$-equivalence, that is, it is a property determined by the $S$-matrix of the Drinfeld center; see Theorem 6.3.

The paper is organized as follows. Section 2 contains the main notions and facts on fusion categories that will be needed in the rest of the paper. In Section 3 we study the notion of Grothendieck equivalence of fusion categories and its connection with solvability, and prove some results on the fusion rules of dihedral and symmetric groups. In Section 4 we consider examples of non-solvable fusion categories arising from exact factorizations of the symmetric and alternating groups. The case of braided fusion categories is studied in Section 5 . Finally, in Section 6 we study the notion of $S$-equivalence of spherical fusion categories.

\section{Preliminaries}

The category of finite dimensional vector spaces over $k$ will be denoted by Vect. A fusion category over $k$ is a semisimple rigid monoidal category over $k$ with finitely many isomorphism classes of simple objects, finite-dimensional Hom spaces, and 
such that the unit object $\mathbf{1}$ is simple. Unless otherwise stated, all tensor categories will be assumed to be strict. We refer the reader to [11, [9], for the main notions on fusion categories used throughout.

2.1. Fusion categories. Let $\mathcal{C}$ be a fusion category over $k$. The Grothendieck group $K_{0}(\mathcal{C})$ is a free abelian group with basis $\operatorname{Irr}(\mathcal{C})$ consisting of isomorphism classes of simple objects of $\mathcal{C}$. For an object $X$ of $\mathcal{C}$, let us denote by $[X]$ its class in $K_{0}(\mathcal{C})$.

The tensor product of $\mathcal{C}$ endows $K_{0}(\mathcal{C})$ with a ring structure with unit element $[\mathbf{1}]$ and such that, for all objects $X$ and $Y$ of $\mathcal{C},[X][Y]=[X \otimes Y]$. Let $X, Y \in \operatorname{Irr}(\mathcal{C})$. Then one can write

$$
X Y=\sum_{Z \in \operatorname{Irr}(\mathcal{C})} N_{X, Y}^{Z} Z
$$

where $N_{X, Y}^{Z}$ are non-negative integers, for all $X, Y, Z \in \operatorname{Irr}(\mathcal{C})$. The collection of numbers $\left\{N_{X, Y}^{Z}\right\}_{X, Y, Z}$ are called the fusion rules of $\mathcal{C}$ and they determine the ring structure of $K_{0}(\mathcal{C})$. They are given by the formula

$$
N_{X, Y}^{Z}=\operatorname{dim} \operatorname{Hom}_{\mathcal{C}}(Z, X \otimes Y),
$$

for all $X, Y, Z \in \operatorname{Irr}(\mathcal{C})$. In the terminology of [14, Subsection 2.1], the pair $\left(K_{0}(\mathcal{C}), \operatorname{Irr}(\mathcal{C})\right)$ is a unital based ring.

A fusion subcategory of $\mathcal{C}$ is a full tensor subcategory $\mathcal{D}$ such that $\mathcal{D}$ is replete and stable under direct summands. Fusion subcategories of $\mathcal{C}$ are in bijective correspondence with subrings of $K_{0}(\mathcal{C})$ spanned by a subset of $\operatorname{Irr}(\mathcal{C})$, that is, based subrings of $K_{0}(\mathcal{C})$.

The Frobenius-Perron dimension of a simple object $X \in \mathcal{C}$ is, by definition, the Frobenius-Perron eigenvalue of the matrix of left multiplication by the class of $X$ in the basis $\operatorname{Irr}(\mathcal{C})$ of the Grothendieck ring of $\mathcal{C}$ consisting of isomorphism classes of simple objects. The Frobenius-Perron dimension of $\mathcal{C}$ is the number $\operatorname{FPdim} \mathcal{C}=\sum_{X \in \operatorname{Irr}(\mathcal{C})}(\mathrm{FPdim} X)^{2}$.

We shall indicate by $\operatorname{cd}(\mathcal{C})$ the set of Frobenius-Perron dimensions of simple objects of $\mathcal{C}$. If $1=d_{0}, d_{1}, \ldots, d_{r}$ are distinct positive real numbers and $n_{1}, \ldots, n_{r}$ are natural numbers, we shall say that $\mathcal{C}$ is of type $\left(d_{0}, n_{0} ; d_{1}, n_{1} ; \ldots ; d_{r}, n_{r}\right)$ if $\mathcal{C}$ has $n_{i}$ isomorphism classes of simple objects of Frobenius-Perron dimension $d_{i}$, for all $i=0, \ldots, r$.

The group of invertible objects of $\mathcal{C}$ will be denoted by $G(\mathcal{C})$. Thus $G(\mathcal{C})$ coincides with the subset of elements $Y$ of $\operatorname{Irr}(\mathcal{C})$ such that FPdim $Y=1$. Thus, if $\mathcal{C}$ is of type $\left(1, n_{0} ; d_{1}, n_{1} ; \ldots ; d_{r}, n_{r}\right)$, then $n_{0}=|G(\mathcal{C})|$.

The category $\mathcal{C}$ is called integral if $\mathrm{FPdim} X \in \mathbb{Z}$, for all simple object $X \in \mathcal{C}$, and it is called weakly integral if $\operatorname{FPdim} \mathcal{C} \in \mathbb{Z}$.

Recall that a right module category over a fusion category $\mathcal{C}$ is a finite semisimple $k$-linear abelian category $\mathcal{M}$ endowed with a bifunctor $\otimes: \mathcal{M} \times \mathcal{C} \rightarrow \mathcal{M}$ satisfying the associativity and unit axioms for an action, up to coherent natural isomorphisms. The module category $\mathcal{M}$ is called indecomposable if it is not equivalent as a module category to a direct sum of non-trivial module categories. If $\mathcal{M}$ is an indecomposable module category over $\mathcal{C}$, then the category $\mathcal{C}_{\mathcal{M}}^{*}$ of $\mathcal{C}$-module endofunctors of $\mathcal{M}$ is also a fusion category.

Two fusion categories $\mathcal{C}$ and $\mathcal{D}$ are Morita equivalent if $\mathcal{D}$ is equivalent to $\mathcal{C}_{\mathcal{M}}^{*}$ for some indecomposable module category $\mathcal{M}$. If $\mathcal{C}$ and $\mathcal{D}$ are Morita equivalent fusion categories, then $\mathrm{FP} \operatorname{dim} \mathcal{C}=\mathrm{FPdim} \mathcal{D}$.

By [12, Theorem 3.1], the fusion categories $\mathcal{C}$ and $\mathcal{D}$ are Morita equivalent if and only if its Drinfeld centers are equivalent as braided fusion categories. 
A fusion category $\mathcal{C}$ is pointed if all its simple objects are invertible. If $\mathcal{C}$ is a pointed fusion category, then there exist a finite group $G$ and a 3-cocycle $\omega$ on $G$ such that $\mathcal{C}$ is equivalent to the category $\mathcal{C}(G, \omega)$ of finite-dimensional $G$-graded vector spaces with associativity constraint defined by $\omega$. A fusion category Morita equivalent to a pointed fusion category is called group-theoretical.

2.2. Nilpotent and solvable fusion categories. Let $G$ be a finite group. A $G$ grading on a fusion category $\mathcal{C}$ is a decomposition $\mathcal{C}=\oplus_{g \in G} \mathcal{C}_{g}$, such that $\mathcal{C}_{g} \otimes \mathcal{C}_{h} \subseteq$ $\mathcal{C}_{g h}$ and $\mathcal{C}_{g}^{*} \subseteq \mathcal{C}_{g^{-1}}$, for all $g, h \in G$. A $G$-grading is faithful if $\mathcal{C}_{g} \neq 0$, for all $g \in G$. The fusion category $\mathcal{C}$ is called a $G$-extension of a fusion category $\mathcal{D}$ if there is a faithful grading $\mathcal{C}=\oplus_{g \in G} \mathcal{C}_{g}$ with neutral component $\mathcal{C}_{e} \cong \mathcal{D}$.

If $\mathcal{C}$ is any fusion category, there exist a finite group $U(\mathcal{C})$, called the universal grading group of $\mathcal{C}$, and a canonical faithful grading $\mathcal{C}=\oplus_{g \in U(\mathcal{C})} \mathcal{C}_{g}$, with neutral component $\mathcal{C}_{e}=\mathcal{C}_{a d}$, where $\mathcal{C}_{a d}$ is the adjoint subcategory of $\mathcal{C}$, that is, the fusion subcategory generated by $X \otimes X^{*}, X \in \operatorname{Irr}(\mathcal{C})$.

In fact, $K_{0}(\mathcal{C})_{a d}=K_{0}\left(\mathcal{C}_{a d}\right)$ is a based subring of $K_{0}(\mathcal{C})$ and $K_{0}(\mathcal{C})$ decomposes into a direct sum of indecomposable based $K_{0}(\mathcal{C})_{a d}$-bimodules $K_{0}(\mathcal{C})=$ $\oplus_{g \in U(\mathcal{C})} K_{0}(\mathcal{C})_{g}$, with $K_{0}(\mathcal{C})_{e}=K_{0}(\mathcal{C})_{a d}$. Then the group structure on $U(\mathcal{C}):=$ $U\left(K_{0}(\mathcal{C})\right)$ is defined by the following property: $g h=t$ if and only if $X_{g} X_{h} \in K_{0}(\mathcal{C})_{t}$, for all $X_{g} \in K_{0}(\mathcal{C})_{g}, X_{h} \in K_{0}(\mathcal{C})_{h}, g, h, t \in U(\mathcal{C})$; see [14, Theorem 3.5].

A fusion category $\mathcal{C}$ is (cyclically) nilpotent if there exists a sequence of fusion categories Vect $=\mathcal{C}_{0} \subseteq \mathcal{C}_{1} \cdots \subseteq \mathcal{C}_{n}=\mathcal{C}$, and finite (cyclic) groups $G_{1}, \ldots, G_{n}$, such that for all $i=1, \ldots, n, \mathcal{C}_{i}$ is a $G_{i}$-extension of $\mathcal{C}_{i-1}$.

On the other side, $\mathcal{C}$ is solvable if it is Morita equivalent to a cyclically nilpotent fusion category, that is, if there exists a cyclically nilpotent fusion category $\mathcal{D}$ and an idecomposable right module category $\mathcal{M}$ over $\mathcal{D}$ such that $\mathcal{C}$ is equivalent to the fusion category $\mathcal{D}_{\mathcal{M}}^{*}$ of $\mathcal{D}$-linear endofunctors of $\mathcal{M}$.

Consider an action of a finite group $G$ on a fusion category $\mathcal{C}$ by tensor autoequivalences $\rho: \underline{G} \rightarrow \underline{\mathrm{Aut}}_{\otimes} \mathcal{C}$. The equivariantization of $\mathcal{C}$ with respect to the action $\rho$, denoted $\mathcal{C}^{G}$, is a fusion category whose objects are pairs $(X, \mu)$, such that $X$ is an object of $\mathcal{C}$ and $\mu=\left(\mu^{g}\right)_{g \in G}$, is a collection of isomorphisms $\mu^{g}: \rho^{g} X \rightarrow X$, $g \in G$, satisfying appropriate compatibility conditions.

The forgetful functor $F: \mathcal{C}^{G} \rightarrow \mathcal{C}, F(X, \mu)=X$, is a dominant tensor functor that gives rise to a central exact sequence of fusion categories $\operatorname{Rep} G \rightarrow \mathcal{C}^{G} \rightarrow \mathcal{C}$ 4], where $\operatorname{Rep} G$ is the category of finite-dimensional representations of $G$.

The category $\mathcal{C}^{G}$ is integral (respectively, weakly integral) if and only if so is $\mathcal{C}$. See [3, Proposition 4.9], 4, Proposition 2.12].

According to [12, Definition 1.2], a fusion category $\mathcal{C}$ is solvable if and only if there exists a sequence of fusion categories Vect $=\mathcal{C}_{0}, \ldots, \mathcal{C}_{n}=\mathcal{C}, n \geq 0$, and a sequence of cyclic groups of prime order $G_{1}, \ldots, G_{n}$, such that, for all $1 \leq i \leq n$, $\mathcal{C}_{i}$ is a $G_{i}$-equivariantization or a $G_{i}$-extension of $\mathcal{C}_{i-1}$.

It is shown in [12, Proposition 4.1] that the class of solvable fusion categories is stable under taking extensions and equivariantizations by solvable groups, Morita equivalent categories, tensor products, Drinfeld center, fusion subcategories and components of quotient categories.

In view of [12, Proposition 4.5 (iv)], every nontrivial solvable fusion category has nontrivial invertible objects.

Suppose that the finite group $G$ acts on the fusion category $\mathcal{C}$ by tensor autoequivalences. Let $Y \in \operatorname{Irr} \mathcal{C}$. The stabilizer of $Y$ is the subgroup $G_{Y}=\{g \in G$ : $\left.\rho^{g}(Y) \cong Y\right\}$. Let $\alpha_{Y}: G_{Y} \times G_{Y} \rightarrow k^{*}$ be the 2-cocycle defined by the relation

$$
\alpha_{Y}(g, h)^{-1} \operatorname{id}_{Y}=c^{g} \rho^{g}\left(c^{h}\right)\left(\rho_{2_{Y}}^{g, h}\right)^{-1}\left(c^{g h}\right)^{-1}: Y \rightarrow Y,
$$


where, for all $g \in G_{Y}, c^{g}: \rho^{g}(Y) \rightarrow Y$ is a fixed isomorphism [5, Subsection 2.3].

Then the simple objects of $\mathcal{C}^{G}$ are parameterized by pairs $(Y, U)$, where $Y$ runs over the $G$-orbits on $\operatorname{Irr}(\mathcal{C})$ and $U$ is an equivalence class of an irreducible $\alpha_{Y^{-}}$ projective representation of $G_{Y}$. We shall use the notation $S_{Y, U}$ to indicate the isomorphism class of the simple object corresponding to the pair $(Y, U)$. The dimension of $S_{Y, U}$ is given by the formula

$$
\operatorname{FPdim} S_{Y, U}=\left[G: G_{Y}\right] \operatorname{dim} U \mathrm{FPdim} Y .
$$

Lemma 2.1. Let $p$ be a prime number. Suppose that the group $\mathbb{Z}_{p}$ acts on a fusion category $\mathcal{C}$ by tensor autoequivalences. Assume in addition that $G\left(\mathcal{C}^{\mathbb{Z}_{p}}\right)$ is of order $p$ and $G(\mathcal{C}) \neq\{1\}$. Then $\mathcal{C}^{\mathbb{Z}_{p}}$ has a simple object of Frobenius-Perron dimension $p$.

Proof. Let $Y$ be an invertible object of $\mathcal{C}$ and let $U$ be an irreducible $\alpha_{Y}$-projective representation of the subgroup $G_{Y} \subseteq \mathbb{Z}_{p}$. Since $G_{Y}$ is cyclic, then $\alpha_{Y}=1$ in $H^{2}\left(G_{Y}, k^{*}\right)$ and $\operatorname{dim} U=1$. Then the Frobenius-Perron dimension of the simple object $S_{Y, U}$ is given by $\mathrm{FPdim} S_{Y, U}=\left[G: G_{Y}\right] \mathrm{FP} \operatorname{dim} Y=\left[G: G_{Y}\right]$. Moreover, if $Y=\mathbf{1}$, then $G_{Y}=\mathbb{Z}_{p}$. Therefore, letting $U_{0}=\epsilon, U_{1}, \ldots, U_{p-1}$ the non-isomorphic representations of $\mathbb{Z}_{p}$, we get that $\mathbf{1}=S_{\mathbf{1}, U_{0}}, S_{\mathbf{1}, U_{1}}, \ldots, S_{\mathbf{1}, U_{p-1}}$ are all the nonisomorphic invertible objects of $\mathcal{C}^{\mathbb{Z}_{p}}$. Hence for all invertible object $Y \neq \mathbf{1}$ of $\mathcal{C}$, we must have $\left[G: G_{Y}\right]=p$ and the simple object $S_{Y, U}$ has Frobenius-Perron dimension $p$. This proves the lemma.

Proposition 2.2. Let $p$ be a prime number. Suppose that $\mathcal{C}$ is a solvable fusion category such that $G(\mathcal{C}) \cong \mathbb{Z}_{p}$ and $\mathcal{C}$ has no simple objects of Frobenius-Perron dimension $p$. Then $\mathcal{C}$ is cyclically nilpotent.

Proof. The proof is by induction on $\mathrm{FPdim} \mathcal{C} \geq p$. If $\mathrm{FPdim} \mathcal{C}=p$ there is nothing to prove. Suppose FPdim $\mathcal{C}>p$. Since $\mathcal{C}$ is solvable then, for some prime number $q$, $\mathcal{C}$ must be a $\mathbb{Z}_{q}$-extension or a $\mathbb{Z}_{q}$-equivariantization of a fusion category $\mathcal{D}$. If the second possibility holds, then the assumption that $G(\mathcal{C}) \cong \mathbb{Z}_{p}$ implies that $q=p$. Moreover, since $\mathcal{D}$ is also solvable, then $\mathcal{D}_{p t} \neq$ Vect. By Lemma 2.1, $\mathcal{C}$ must have a simple object of dimension $p$, which contradicts the assumption.

Therefore $\mathcal{C}$ must be a $\mathbb{Z}_{q}$-extension of a fusion subcategory $\mathcal{D}$. In particular, $\mathcal{D}$ cannot have simple objects of dimension $p$ and since $\mathcal{D}$ is solvable, then $\mathcal{D}_{p t} \neq$ Vect, whence $G(\mathcal{D})=G(\mathcal{C}) \cong \mathbb{Z}_{p}$. By induction, $\mathcal{D}$ and then also $\mathcal{C}$, is cyclically nilpotent. This finishes the proof of the proposition.

Lemma 2.3. Let $\mathcal{C}$ be a fusion category and let $G$ be a finite group acting on $\mathcal{C}$ by tensor autoequivalences. Then the forgetful functor $U: \mathcal{Z}\left(\mathcal{C}^{G}\right) \rightarrow \mathcal{C}^{G}$ induces an injective ring homomorphism $K_{0}(G) \rightarrow Z\left(K_{0}\left(\mathcal{C}^{G}\right)\right)$. In particular, the group $\widehat{G}$ is isomorphic to a subgroup of the center of $G\left(\mathcal{C}^{G}\right)$.

Proof. By [12, Proposition 2.10], the Drinfeld center $\mathcal{Z}(\mathcal{C})$ contains a Tannakian subcategory $\mathcal{E} \cong \operatorname{Rep} G$ such that $\mathcal{E}$ embeds into $\mathcal{C}$ under the forgetful functor $U: \mathcal{Z}(\mathcal{C}) \rightarrow \mathcal{C}$. As a consequence we obtain the lemma.

2.3. Braided fusion categories. A braided fusion category is a fusion category $\mathcal{C}$ endowed with a braiding, that is, a natural isomorphism $c_{X, Y}: X \otimes Y \rightarrow Y \otimes X$, $X, Y \in \mathcal{C}$, subject to the so-called hexagon axioms.

If $\mathcal{D}$ is a fusion subcategory of a braided fusion category $\mathcal{C}$, the Müger centralizer of $\mathcal{D}$ in $\mathcal{C}$ will be denoted by $\mathcal{D}^{\prime}$. Thus $\mathcal{D}^{\prime}$ is the full fusion subcategory generated by all objects $X \in \mathcal{C}$ such that $c_{Y, X} c_{X, Y}=\operatorname{id}_{X \otimes Y}$, for all objects $Y \in \mathcal{D}$. 
The centralizer $\mathcal{C}^{\prime}$ of $\mathcal{C}$ is called the Müger (or symmetric) center of $\mathcal{C}$. The category $\mathcal{C}$ is called symmetric if $\mathcal{C}^{\prime}=\mathcal{C}$. If $\mathcal{C}$ is any braided fusion category, its Müger center $\mathcal{C}^{\prime}$ is a symmetric fusion subcategory of $\mathcal{C}$. The category $\mathcal{C}$ is called non-degenerate (respectively, slightly degenerate) if $\mathcal{C}^{\prime} \cong$ Vect (respectively, if $\mathcal{C}^{\prime} \cong$ sVect, where sVect denotes the category of super-vector spaces).

For a fusion category $\mathcal{C}$, the Drinfeld center of $\mathcal{C}$ will be denoted $\mathcal{Z}(\mathcal{C})$. It is known that $\mathcal{Z}(\mathcal{C})$ is a braided non-degenerate fusion category of Frobenius-Perron $\operatorname{dimension} \operatorname{FPdim} \mathcal{Z}(\mathcal{C})=(\operatorname{FPdim} \mathcal{C})^{2}$.

Let $G$ be a finite group. The fusion category $\operatorname{Rep} G$ of finite dimensional representations of $G$ is a symmetric fusion category with respect to the canonical braiding. A braided fusion category $\mathcal{E}$ is called Tannakian, if $\mathcal{E} \cong \operatorname{Rep} G$ for some finite group $G$ as braided fusion categories.

Every symmetric fusion category is equivalent, as a braided fusion category, to the category $\operatorname{Rep}(G, u)$ of representations of a finite group $G$ on finite-dimensional super-vector spaces, where $u \in G$ is a central element of order 2 which acts as the parity operator [6]. In particular, if $\mathcal{C}$ is symmetric, then it is equivalent to the category of representations of a finite group as a fusion category.

Let $G$ be a finite group. A $G$-crossed braided fusion category is a fusion category $\mathcal{D}$ endowed with a $G$-grading $\mathcal{D}=\oplus_{g \in G} \mathcal{D}_{g}$ and an action of $G$ by tensor autoequivalences $\rho: \underline{G} \rightarrow \underline{\text { Aut }} \otimes \mathcal{D}$, such that $\rho^{g}\left(\mathcal{D}_{h}\right) \subseteq \mathcal{D}_{g h g^{-1}}$, for all $g, h \in G$, and a $G$-braiding $c: X \otimes Y \rightarrow \rho^{g}(Y) \otimes X, g \in G, X \in \mathcal{D}_{g}, Y \in \mathcal{D}$, subject to compatibility conditions. The $G$-braiding $c$ restricts to a braiding in the neutral component $\mathcal{D}_{e}$.

If $\mathcal{D}$ is a $G$-crossed braided fusion category, then the equivariantization $\mathcal{D}^{G}$ under the action of $G$ is a braided fusion category containing $\operatorname{Rep} G$ as a Tannakian subcategory. Furthermore, the group $G$ acts by restriction on $\mathcal{D}_{e}$ by braided tensor autoequivalences. The equivariantization $\mathcal{D}_{e}^{G}$ coincides with the centralizer $\mathcal{E}^{\prime}$ of the Tannakian subcategory $\mathcal{E}$ in $\mathcal{D}^{G}$. See [22].

Let $\mathcal{E}$ be Tannakian subcategory of a braided fusion category $\mathcal{C}$ and let $G$ be a finite group such that $\mathcal{E} \cong \operatorname{Rep} G$ as symmetric categories. Let also $A \in \mathcal{C}$ be the algebra corresponding to the algebra $k^{G} \in \operatorname{Rep} G$ of functions on $G$ with the regular action. The de-equivariantization $\mathcal{C}_{G}$ of $\mathcal{C}$ with respect to $\operatorname{Rep} G$ is the fusion category $\mathcal{C}_{A}$ of right $A$-modules in $\mathcal{C}$. This is a $G$-crossed braided fusion category such that $\mathcal{C} \cong\left(\mathcal{C}_{G}\right)^{G}$. The neutral component of $\mathcal{C}_{G}$ with respect to the associated $G$-grading, denoted by $\mathcal{C}_{G}^{0}$, coincides with the de-equivariantization $\mathcal{E}^{\prime}{ }_{G}$ of the centralizer of $\mathcal{E}$ by the group $G$.

It was shown in [29, Proposition ] that if $\mathcal{E} \cong \operatorname{Rep} G \subseteq \mathcal{C}$ is a Tannakian subcategory, then $\mathcal{C}$ is weakly integral (respectively, integral or weakly group-theoretical) if and only if $\mathcal{C}_{G}^{0}$ is weakly integral (respectively, integral, weakly group-theoretical). In addition, $\mathcal{C}$ is solvable if and only if $\mathcal{C}_{G}^{0}$ is solvable and $G$ is solvable.

Lemma 2.4. Let $\mathcal{C}$ be a braided fusion category. Then the subcategory $\mathcal{C}_{a d} \cap \mathcal{C}_{p t}$ is symmetric.

Proof. Suppose first that $\mathcal{C}$ is non-degenerate. Then $\mathcal{C}_{a d}=\mathcal{C}_{p t}^{\prime}$, by [9, Corollary 3.27]. Therefore $\mathcal{C}_{a d} \cap \mathcal{C}_{p t}=\mathcal{C}_{p t}^{\prime} \cap \mathcal{C}_{p t}$ is a symmetric subcategory.

Next, for an arbitrary braided fusion category $\mathcal{C}$, let $\mathcal{Z}(\mathcal{C})$ be the Drinfeld center of $\mathcal{C}$. Since $\mathcal{Z}(\mathcal{C})$ is non-degenerate, then the category $\mathcal{Z}(\mathcal{C})_{a d} \cap \mathcal{Z}(\mathcal{C})_{p t}$ is symmetric. The braiding of $\mathcal{C}$ induces a canonical embedding of braided fusion categories $\mathcal{C} \rightarrow$ $\mathcal{Z}(\mathcal{C})$. We may therefore identify $\mathcal{C}$ with a fusion subcategory of $\mathcal{Z}(\mathcal{C})$. Observe that $\mathcal{C}_{a d} \subseteq \mathcal{Z}(\mathcal{C})_{a d}$ and $\mathcal{C}_{p t} \subseteq \mathcal{Z}(\mathcal{C})_{p t}$. Hence $\mathcal{C}_{a d} \cap \mathcal{C}_{p t} \subseteq \mathcal{Z}(\mathcal{C})_{a d} \cap \mathcal{Z}(\mathcal{C})_{p t}$, and then $\mathcal{C}_{a d} \cap \mathcal{C}_{p t}$ is symmetric, as claimed. 
Lemma 2.5. Let $\mathcal{C}$ be a braided fusion category such that $\mathcal{C}_{a d}=\mathcal{C}$. Then $\mathcal{C}_{p t}^{\prime}=\mathcal{C}$.

Proof. Let $\mathcal{B} \subseteq \mathcal{C}$ be any fusion subcategory. By [9, Proposition 3.25] we have $\left(\mathcal{B}_{a d}\right)^{\prime}=\left(\mathcal{B}^{\prime}\right)^{c o}=\mathcal{A}$, where $\mathcal{A} \subseteq \mathcal{C}$ denotes the projective centralizer of $\mathcal{B}$. Letting $\mathcal{B}=\mathcal{C}_{p t}$ we find that $\mathcal{C}=\left(\mathcal{B}_{a d}\right)^{\prime}$ equals the projective centralizer of $\mathcal{C}_{p t}$. By [9. Lemma 3.15], the projective centralizer of a fusion subcategory $\mathcal{B}$ is a graded extension of the centralizer $\mathcal{B}^{\prime}$. Since $\mathcal{C}=\mathcal{C}_{a d}$, this implies that $\mathcal{C}=\mathcal{C}_{p t}^{\prime}$, as claimed.

\section{Grothendieck equivalence of Fusion CAtegories}

Let $\mathcal{C}$ and $\tilde{\mathcal{C}}$ be fusion categories. A Grothendieck equivalence between $\mathcal{C}$ and $\tilde{\mathcal{C}}$ is a bijection $f: \operatorname{Irr} \mathcal{C} \rightarrow \operatorname{Irr} \tilde{\mathcal{C}}$ such that

$$
f(\mathbf{1})=\mathbf{1}, \quad \text { and } N_{f(X), f(Y)}^{f(Z)}=N_{X, Y}^{Z},
$$

for all $X, Y, Z \in \operatorname{Irr} \mathcal{C}$.

We shall say that $\mathcal{C}$ and $\tilde{\mathcal{C}}$ are Grothendieck equivalent if there exist a Grothendieck equivalence between them.

Remark 3.1. Suppose $f: \operatorname{Irr} \mathcal{C} \rightarrow \operatorname{Irr} \tilde{\mathcal{C}}$ is a Grothendieck equivalence. Then the map $f$ extends canonically to a ring isomorphism $f: K_{0}(\mathcal{C}) \rightarrow K_{0}(\tilde{\mathcal{C}})$.

In particular, $f$ induces a bijection between the lattices of fusion subcategories of $\mathcal{C}$ and $\tilde{\mathcal{C}}$. If $\mathcal{D}$ is a fusion subcategory of $\mathcal{C}$, we shall denote by $f(\mathcal{D})$ the corresponding fusion subcategory of $\tilde{\mathcal{C}}$, that is, $f(\mathcal{D})$ is the fusion subcategory whose simple objects are $f(X), X \in \operatorname{Irr} \mathcal{D}$. Note that $f$ restricts to a Grothendieck equivalence $f$ : $\operatorname{Irr} \mathcal{D} \rightarrow \operatorname{Irr} f(\mathcal{D})$.

Proposition 3.2. Let $\mathcal{C}$ and $\tilde{\mathcal{C}}$ be fusion categories and suppose that $f: \operatorname{Irr} \mathcal{C} \rightarrow \operatorname{Irr} \tilde{\mathcal{C}}$ is a Grothendieck equivalence. Then the following hold:

(i) If $X \in K_{0}(\mathcal{C})$, then $\operatorname{FPdim}(f(X))=\mathrm{FPdim}(X)$. Hence, if $\mathcal{D}$ is a fusion subcategory of $\mathcal{C}$, then $\operatorname{FPdim}(f(\mathcal{D}))=\operatorname{FPdim}(\mathcal{D})$.

(ii) $X \in \operatorname{Irr} \mathcal{C}$ is invertible if and only if $f(X) \in \operatorname{Irr} \tilde{\mathcal{C}}$ is invertible.

(iii) If $X \in \operatorname{Irr} \mathcal{C}$, then $f\left(X^{*}\right)=f(X)^{*}$.

(iv) $f\left(\mathcal{C}^{(n)}\right)=\tilde{\mathcal{C}}^{(n)}$, for all $n \geq 0$. In particular, $\mathcal{C}$ is nilpotent if and only if $\tilde{\mathcal{C}}$ is nilpotent.

(v) $f$ induces a group isomorphism $f: U(\mathcal{C}) \rightarrow U(\tilde{\mathcal{C}})$ such that $f\left(\mathcal{C}_{g}\right)=\tilde{\mathcal{C}}_{f(g)}$.

Proof. (i) By Remark 3.1 we know that $f$ extends to a ring isomorphism $f$ : $K_{0}(\mathcal{C}) \rightarrow K_{0}(\tilde{\mathcal{C}})$. By [11, Lemma 8.3] FPdim $: K_{0}(\mathcal{C}) \rightarrow \mathbb{R}$ is the only ring homomorphism such that $\operatorname{FPdim}(X)>0$ for any $0 \neq X \in \mathcal{C}$, so $\operatorname{FPdim}(f(X))=$ $\operatorname{FPdim}(X)$, for all $X \in \operatorname{Irr} \mathcal{C}$.

(ii) This follows from (i), since the invertible objects of a fusion category are exactly those objects with Frobenius-Perron dimension 1.

(iii) Since $f(\mathbf{1})=\mathbf{1}$, then $N_{f(X), f\left(X^{*}\right)}^{1}=N_{X, X^{*}}^{1}=1$. Therefore $f\left(X^{*}\right)=f(X)^{*}$.

(iv) It follows from (iii) and the fact that $f$ preserves fusion rules that $f\left(\mathcal{C}_{a d}\right)=$ $\tilde{\mathcal{C}}_{a d}$. Then $f$ induces by restriction a Grothendieck equivalence $\operatorname{Irr} \mathcal{C}_{a d} \rightarrow \operatorname{Irr} \tilde{\mathcal{C}}_{a d}$. An inductive argument implies that $f\left(\mathcal{C}^{(n)}\right)=\tilde{\mathcal{C}}^{(n)}$, for all $n \geq 0$.

(v) By definition $U(\mathcal{C})=U\left(K_{0}(\mathcal{C})\right)\left[14\right.$ and $K_{0}(\mathcal{C})$ decomposes into a direct sum of indecomposable based $K_{0}(\mathcal{C})_{a d}$-bimodules $K_{0}(\mathcal{C})=\oplus_{g \in U(\mathcal{C})} K_{0}(\mathcal{C})_{g}$, with $K_{0}(\mathcal{C})_{e}=K_{0}(\mathcal{C})_{a d}$. This decomposition is unique up to a permutation of $U(\mathcal{C})$. By Remark 3.1, $f$ extends to a ring isomorphism $f: K_{0}(\mathcal{C}) \rightarrow K_{0}(\tilde{\mathcal{C}})$ and by (iv) $f$ restricts to a ring isomorphism $K_{0}(\mathcal{C})_{a d}=K_{0}\left(\mathcal{C}_{a d}\right) \cong K_{0}\left(\tilde{\mathcal{C}}_{a d}\right)=K_{0}(\tilde{\mathcal{C}})_{a d}$. So for all $g \in U(\mathcal{C}), f\left(K_{0}(\mathcal{C})_{g}\right)=K_{0}(\tilde{\mathcal{C}})_{\tilde{g}}$, for a unique $\tilde{g} \in U(\tilde{\mathcal{C}})$. Letting $f(g)=\tilde{g}$, we obtain a group isomorphism $f: U(\mathcal{C}) \rightarrow U(\tilde{\mathcal{C}})$ such that $f\left(K_{0}\left(\mathcal{C}_{g}\right)\right)=K_{0}\left(\tilde{\mathcal{C}}_{f(g)}\right)$. This implies (v). 
Remark 3.3. Let $G$ be a finite group. Observe that any $G$-grading on a fusion category $\mathcal{C}$ with neutral component $\mathcal{D}$ is uniquely determined by a $G$-grading on the Grothendieck ring $K_{0}(\mathcal{C})$ with neutral component $K_{0}(\mathcal{D})$. In particular, if $\mathcal{C}$ and $\tilde{\mathcal{C}}$ are Grothendieck equivalent, then $\mathcal{C}$ is $G$-graded with neutral component $\mathcal{D}$ if and only if $\tilde{\mathcal{C}}$ is $G$-graded with neutral component $\tilde{\mathcal{D}}$, such that $\tilde{\mathcal{D}}$ and $\mathcal{D}$ are Grothendieck equivalent.

Our first theorem concerns fusion categories with dihedral fusion rules.

Theorem 3.4. Let $n$ be a natural number and let $\mathcal{C}$ be a fusion category. Suppose that $\mathcal{C}$ is Grothendieck equivalent to the category $\operatorname{Rep} D_{n}$, where $D_{n}$ is the dihedral group of order $2 n$. Then $\mathcal{C}$ is solvable.

Proof. It follows from [24, Theorem 4.2] that a fusion category Grothendieck equivalent to the representation category of a dihedral group is group-theoretical. Then $\mathcal{C}$ is group-theoretical, that is, it is Morita equivalent to a pointed fusion category $\mathcal{C}(\Gamma, \omega)$, where $\Gamma$ is a group and $\omega$ is a 3-cocycle on $\Gamma$.

Suppose first that $n$ is odd. Then the order of $\Gamma$ is equal to $2 n$ and, since $n$ is odd, $\Gamma$ is solvable. Then $\mathcal{C}$ is solvable too.

If $n$ is even, then the center of $D_{n}$ is of order 2 and $D_{n} / Z\left(D_{n}\right) \cong D_{n / 2}$. Therefore, the category $\operatorname{Rep} D_{n}$ is a $\mathbb{Z}_{2}$-extension of Rep $D_{n / 2}$; see [14, Example 3.2]. Since $\mathcal{C}$ is Grothendieck equivalent to $\operatorname{Rep} D_{n}$, then it is a $\mathbb{Z}_{2}$-extension of a fusion subcategory $\mathcal{D}_{1}$, where $\mathcal{D}_{1}$ is Grothendieck equivalent to $\operatorname{Rep} D_{n / 2}$. Continuing this process, we find that the category $\mathcal{C}$ is obtained by a sequence of $\mathbb{Z}_{2}$-extensions from a fusion subcategory $\mathcal{D}$ such that $\mathcal{D}$ is Grothendieck equivalent to $\operatorname{Rep} D_{m}$, with $m$ an odd natural number. By the above, $\mathcal{D}$ is solvable and therefore so is $\mathcal{C}$. This finishes the proof of the theorem.

The following consequence of Proposition 2.2 gives some restrictions that guarantee that the solvability of a fusion category is a Grothendieck invariant.

Proposition 3.5. Let $p$ be a prime number. Suppose that $\mathcal{C}$ is a solvable fusion category such that $G(\mathcal{C}) \cong \mathbb{Z}_{p}$ and $\mathcal{C}$ has no simple objects of Frobenius-Perron dimension $p$. If $\mathcal{C}$ is Grothendieck equivalent to a fusion category $\tilde{\mathcal{C}}$, then $\tilde{\mathcal{C}}$ is solvable.

Proof. By Proposition 2.2, $\mathcal{C}$ is cyclically nilpotent. Therefore $\tilde{\mathcal{C}}$ is cyclically nilpotent, whence solvable.

Remark 3.6. For all $n \geq 2$, the alternating group $\mathbb{A}_{n}$ has no irredubible representation of degree 21. In addition, if $n \geq 5\left(\operatorname{Rep} \mathbb{S}_{4}\right.$ is of type $\left.(1,2 ; 2,1 ; 3,2)\right)$, the symmetric group $\mathbb{S}_{n}$ has no irredubible representation of degree 2 neither. In fact, if $V$ were such a representation, then the restriction $\left.V\right|_{\mathbb{A}_{n}}$ would not be irreducible. Hence, since $\mathbb{A}_{n}$ has no nontrivial one-dimensional representations (because $n \geq 5$ ), then $\left.V\right|_{\mathbb{A}_{n}}$ would be trivial. This is impossible, because the kernel of the restriction functor Rep $\mathbb{S}_{n} \rightarrow \operatorname{Rep} \mathbb{A}_{n}$ is the pointed subcategory Rep $\mathbb{Z}_{2} \subseteq \operatorname{Rep} \mathbb{S}_{n}$.

Corollary 3.7. Let $n \geq 5$ be a natural number and let $\mathcal{C}$ be a fusion category. Suppose that $\mathcal{C}$ is Grothendieck equivalent to $\operatorname{Rep} \mathbb{S}_{n}$. Then $\mathcal{C}$ is not solvable.

Proof. The category Rep $\mathbb{S}_{n}$ is not solvable. On the other hand, the group $\mathbb{S}_{n}$ has two non-equivalent representations of degree one and no irreducible representation of degree two, in view of Remark 3.6. Hence $G(\mathcal{C}) \cong \mathbb{Z}_{2}$ and $\mathcal{C}$ has no simple objects of Frobenius-Perron dimension 2. The result is thus obtained as a consequence of Proposition 3.5 .

\footnotetext{
${ }^{1}$ This can be seen, for instance, as a consequence of the Nichols-Richmond theorem 30, Theorem 11]
} 
Remark 3.8. Let $G$ be a non-abelian finite simple group. If $\mathcal{C}$ is a fusion category Grothendieck equivalent to $\operatorname{Rep} G$, then $\mathcal{C}_{p t}=$ Vect and therefore $\mathcal{C}$ is not solvable.

\section{EXAmples of NON-SOlVABle FUSiOn RULES}

4.1. Abelian extensions. Consider an abelian exact sequence of Hopf algebras

$$
k \longrightarrow k^{\Gamma} \stackrel{i}{\longrightarrow} H \stackrel{\pi}{\longrightarrow} k F \longrightarrow k,
$$

where $\Gamma$ and $F$ are finite groups. Then (4.1) gives rise to actions by permutations $\Gamma \longleftarrow \Gamma \times F \stackrel{\triangleright}{\longleftarrow} F$ such that $(\Gamma, F)$ is a matched pair of groups. Moreover, $H \cong k^{\Gamma \tau} \#_{\sigma} k F$ is a bicrossed product with respect to normalized invertible 2cocycles $\sigma: F \times F \rightarrow k^{\Gamma}, \tau: \Gamma \times \Gamma \rightarrow k^{F}$, satisfying suitable compatibility conditions. See 17 .

The multiplication and comultiplication of $k^{\Gamma \tau} \#_{\sigma} k F$ are determined in the basis $\left\{e_{s} \# x / s \in \Gamma, x \in F\right\}$, by the formulas

$$
\begin{aligned}
\left(e_{s} \# x\right) \otimes\left(e_{t} \# y\right) & =\delta_{t, s \triangleleft x} \sigma_{s}(x, y) e_{s} \# x y, \\
\Delta\left(e_{s} \# x\right) & =\sum_{g h=s} \tau_{x}(g, h) e_{g} \#(h \triangleright x) \otimes e_{h} \# x,
\end{aligned}
$$

for all $s, t \in \Gamma, x, y \in F$, where $\sigma_{s}(x, y)=\sigma(x, y)(s)$ and $\tau_{x}(s, t)=\tau(s, t)(x)$. See 17. The exact sequence (4.1) is called split if $\sigma$ and $\tau$ are the trivial 2-cocycles.

For all $s \in \Gamma$, the restriction of the map $\sigma_{s}: F \times F \rightarrow k^{\times}$to the stabilizer subgroup $F_{s}=F \cap s F s^{-1}$ is a 2-cocycle on $F_{s}$.

The irreducible representations of $H \cong k^{\Gamma \tau} \#_{\sigma} k F$ are classified for pairs $\left(s, U_{s}\right)$, where $s$ is a representative of the orbits of the action of $F$ in $\Gamma$ and $U_{s}$ is an irreducible representation of the twisted group algebra $k_{\sigma_{s}} F_{s}$, that is, a projective irreducible representation $F_{s}$ with cocycle $\sigma_{s}$. Given a pair $\left(s, U_{s}\right)$, the corresponding irreducible representation is given by

$$
W_{\left(s, U_{s}\right)}=\operatorname{Ind}_{k^{\Gamma} \otimes k F_{s}}^{H} s \otimes U_{s} .
$$

Observe that $\operatorname{dim} W_{\left(s, U_{s}\right)}=\left[F: F_{s}\right] \operatorname{dim} U_{s}$. See [20].

Remark 4.1. Recall that every matched pair $(\Gamma, F)$ gives rise to a group structure, denoted $F \bowtie \Gamma$, on the product $F \times \Gamma$ in the form

$$
(x, s)(y, t)=(x(s \triangleright y),(s \triangleleft y) t),
$$

$x, y \in F, s, t \in \Gamma$, where $\Gamma \stackrel{\triangleleft}{\longleftarrow} \Gamma \times F \stackrel{\triangleright}{\longrightarrow} F$ are the associated compatible actions.

The group $F \bowtie \Gamma$ has a canonical exact factorization into its subgroups $F=$ $F \times\{e\}$ and $\Gamma=\{e\} \times \Gamma$; that is, $F \bowtie \Gamma=F \Gamma$ and $F \cap \Gamma=\{e\}$.

Conversely, every finite group $G$ endowed with an exact factorization $G=F \Gamma$ into its subgroups $F$ and $\Gamma$ gives rise to canonical actions by permutations $\Gamma \longleftarrow$ $\Gamma \times F \stackrel{\triangleright}{\longrightarrow} F$ making $(\Gamma, F)$ into a matched pair of groups.

Suppose $H \cong k^{\Gamma \tau} \#_{\sigma} k F$ is an abelian extension of $k^{\Gamma}$ by $k F$. It follows from [25, Theorem 1.3] that the category $\operatorname{Rep} H$ is Morita equivalent to the pointed fusion category $\mathcal{C}(F \bowtie \Gamma, \omega)$, where $\omega$ is a 3-cocycle on $F \bowtie \Gamma$ arising from the pair $(\sigma, \tau)$ in an exact sequence due to G. I. Kac. In particular, there are equivalences of braided fusion categories

$$
\mathcal{Z}(\operatorname{Rep} H) \cong \operatorname{Rep} D(H) \cong \operatorname{Rep} D^{\omega}(F \bowtie \Gamma),
$$

where $D^{\omega}(F \bowtie \Gamma)$ is the twisted Drinfeld double of $F \bowtie \Gamma$ [7]. Note that Rep $H$ is solvable if and only if the group $F \bowtie \Gamma$ is solvable. 
Example 4.2. Let $G$ be a finite group. Then the Drinfeld double $D(G)$ fits into a split cocentral abelian exact sequence

$$
k \longrightarrow k^{G} \longrightarrow D(G) \longrightarrow k G \longrightarrow k .
$$

This exact sequence is associated to the adjoint action $\triangleleft: G \times G \rightarrow G, h \triangleleft g=$ $g^{-1} h g$, and to the trivial action $\triangleright: G \times G \rightarrow G$.

The following lemma describes the group of invertible objects of the category Rep $H$, when $H$ is an abelian extension.

Lemma 4.3. Suppose $H$ fits into an exact sequence (4.1). Then there is an exact sequence

$$
1 \longrightarrow \widehat{F} \stackrel{\pi^{*}}{\longrightarrow} G(\operatorname{Rep} H) \stackrel{i^{*}}{\longrightarrow} \Gamma_{0} \longrightarrow 1,
$$

where $\widehat{F}$ denotes the group of one-dimensional characters of $F$ and $\Gamma_{0}=\left\{s \in \Gamma^{F}\right.$ : $\left[\sigma_{s}\right]=1$ in $\left.H^{2}\left(\Gamma,\left(k^{F}\right)^{\times}\right)\right\}$.

Proof. The group $G(\operatorname{Rep} H)$ can be identified with the group $G\left(H^{*}\right)$ of group-like elements in the dual Hopf algebra $H^{*}$. In addition, $H^{*}$ fits into an abelian extension

$$
k \longrightarrow k^{F} \stackrel{i^{*}}{\longrightarrow} H^{*} \stackrel{\pi^{*}}{\longrightarrow} k \Gamma \longrightarrow k .
$$

The lemma follows from [27, Lemma 2.2].

Remark 4.4. Keep the notation in Lemma 4.3. Note that the dual exact sequence (4.5) is associated to the actions $F \stackrel{\triangleleft^{\prime}}{\longleftarrow} F \times \Gamma \stackrel{\triangleright^{\prime}}{\longrightarrow} \Gamma$ defined in the form $x \triangleleft^{\prime} s=$ $\left(s^{-1} \triangleright x^{-1}\right)^{-1}$ and $x \triangleright^{\prime} s=\left(s^{-1} \triangleleft x^{-1}\right)^{-1}$, for all $x \in F, s \in \Gamma$ [17, Exercise 5.5].

Hence the exact sequence of groups of Lemma 4.3 induces the transpose of the action $\triangleleft^{\prime}$ of $\Gamma_{0}$ on the abelian group $\widehat{F}$.

Clearly, (4.5) is split if and only if (4.1) is split and, if this is the case, the exact sequence of groups in Lemma 4.3 is split as well.

Therefore, in the case where $H$ is a split abelian extension, the group $G(\operatorname{Rep} H)$ is isomorphic to the semidirect product $\widehat{F} \rtimes \Gamma_{0}$ with respect to the action $\triangleleft^{\prime}$ of $\Gamma_{0}$ on $\widehat{F}$.

Corollary 4.5. Let $G$ be a finite group. Then the group of invertible objects of $\operatorname{Rep} D(G)$ is isomorphic to the direct product $G /[G, G] \times Z(G)$.

Proof. This is a consequence of Lemma 4.3, in view of Example 4.2 and Remark 4.4. In fact, $\widehat{G} \cong G /[G, G]$ and the actions $\triangleright^{\prime}: G \times G \rightarrow G$ and $\triangleleft^{\prime}: G \times G \rightarrow G$ in Remark 4.4 are given in this case by $h \triangleright^{\prime} g=h g h^{-1}$ and $g \triangleleft^{\prime} h=g$, for all $g, h \in G$. Then $G_{0}=\left\{g \in G \mid h \triangleright^{\prime} g=g, \forall h \in G\right\}=Z(G)$. The Corollary follows from the fact that the action $\triangleleft^{\prime}$ is the trivial one.

4.2. Examples associated to the symmetric group. Let $n \geq 2$ be a natural number. The symmetric group $\mathbb{S}_{n}$ has an exact factorization $\mathbb{S}_{n}=\langle z\rangle \Gamma$, where $\Gamma=\left\{\sigma \in \mathbb{S}_{n}: \sigma(n)=n\right\} \cong \mathbb{S}_{n-1}$ and $z=(12 \ldots n)$, so that $\langle z\rangle \cong C_{n}$. This exact factorization induces mutual actions by permutations $\mathbb{S}_{n-1} \stackrel{\triangleleft}{\longleftarrow} \mathbb{S}_{n-1} \times C_{n} \stackrel{\triangleright}{\longrightarrow}$ $C_{n}$ that make $\left(\mathbb{S}_{n-1}, C_{n}\right)$ into a matched pair of groups. The actions $\triangleleft, \triangleright$ are determined by the relations

$$
\sigma c=(\sigma \triangleright c)(\sigma \triangleleft c)
$$

for all $\sigma \in \Gamma, c \in\langle z\rangle$.

Suppose $n$ is odd, so that $\langle z\rangle \subseteq \mathbb{A}_{n}$. Relations (4.6) imply that the subgroup $\Gamma_{+}=\Gamma \cap \mathbb{A}_{n} \cong \mathbb{A}_{n-1}$ is stable under the action $\triangleleft$ of $\langle z\rangle$. Therefore the actions $\triangleleft, \triangleright$ induce by restriction a matched pair $\left(\mathbb{A}_{n-1}, C_{n}\right)$. 
Remark 4.6. Let $\sigma \in \Gamma$. It follows from (4.6) that $\sigma z=z^{r}(\sigma \triangleleft z)$, for some $0 \leq r \leq n-1$. Since $\sigma \triangleleft z \in \Gamma$ then $(\sigma \triangleleft z)(n)=n$, implying that $r=b(n)$, where $b=\sigma z$.

Suppose that $n \geq 4$ is even and $\sigma \in \Gamma \cap \mathbb{A}_{n}$. Since $z$ is an odd permutation and $\sigma \triangleleft z=z^{-r} \sigma z$, then $\sigma \triangleleft z$ is even if and only if $r$ is odd. Letting $\sigma=(12 \ldots(n-1)) \in$ $\Gamma \cap \mathbb{A}_{n}$, we find that $r=b(n)=\sigma z(n)=2$; so that $\sigma \triangleleft z$ is an odd permutation. This shows that the subgroup $\Gamma_{+}=\Gamma \cap \mathbb{A}_{n} \cong \mathbb{A}_{n-1}$ is not stable under the action $\triangleleft$ of $\langle z\rangle$ in this case.

Let us consider the associated Hopf algebras $J_{n}=k^{\mathbb{S}_{n-1}} \# k C_{n}$ and $K_{n}=$ $k^{\mathbb{A}_{n-1}} \# k C_{n}$.

The categories Rep $J_{n}$, Rep $K_{n}$ are Morita equivalent to the categories $\operatorname{Rep} \mathbb{S}_{n}$ and $\operatorname{Rep} \mathbb{A}_{n}$, respectively; see Remark 4.1. In particular, $\operatorname{Rep} J_{n}$ and $\operatorname{Rep} K_{n}$ are not solvable, for all $n \geq 5$.

Observe that $J_{n}^{*}$ is a split abelian extension of $k^{C_{n}}$ by $k \mathbb{S}_{n-1}$ associated to the actions $\triangleleft^{\prime}$ and $\triangleright^{\prime}$ in Remark 4.4

Remark 4.7. Suppose $n \geq 5$. It follows from [28, Theorem 5.2], that the Hopf algebras $J_{n}, K_{n}, J_{n}^{*}, K_{n}^{*}$ admit no quasitriangular structure. In particular, the fusion categories $\operatorname{Rep} J_{n}, \operatorname{Rep} K_{n}$, Rep $J_{n}^{*}$ and $\operatorname{Rep} K_{n}^{*}$ admit no braiding.

In addition, there are equivalences of braided fusion categories

$$
\operatorname{Rep} D\left(J_{n}\right) \cong \operatorname{Rep} D\left(\mathbb{S}_{n}\right), \quad \operatorname{Rep} D\left(K_{n}\right) \cong \operatorname{Rep} D\left(\mathbb{A}_{n}\right) .
$$

It follows from Corollary 4.5 that there are group isomorphisms $G\left(D\left(\mathbb{S}_{n}\right)^{*}\right) \cong$ $\mathbb{S}_{n} /\left[\mathbb{S}_{n}, \mathbb{S}_{n}\right] \times Z\left(\mathbb{S}_{n}\right) \cong \mathbb{Z}_{2}$, for all $n \geq 3$, and similarly, $G\left(D\left(\mathbb{A}_{n}\right)^{*}\right)=1$, for all $n \geq 5$.

Lemma 4.8. The pointed subcategory $\operatorname{Rep} D\left(\mathbb{S}_{n}\right)_{p t} \cong \operatorname{Rep} \mathbb{Z}_{2}$ is a Tannakian subcategory of $\operatorname{Rep} D\left(\mathbb{S}_{n}\right)$.

Proof. It follows from the description of the irreducible representations in (4.4), that the one-dimensional representations of $D\left(\mathbb{S}_{n}\right)$ are parameterized by pairs $\left(s, U_{s}\right)$, where $s \in \mathbb{S}_{n}$ is a central element and $U_{s}$ is a one-dimensional representation of $\mathbb{S}_{n}$. Since $Z\left(\mathbb{S}_{n}\right)=\{e\}$ and $\mathbb{S}_{n}$ has two non-isomorphic one dimensional representations trivial one and the sign representation $\operatorname{Sg}$, then $\operatorname{Rep} D\left(\mathbb{S}_{n}\right)_{p t} \cong \operatorname{Rep} \mathbb{Z}_{2}$. Moreover, the unique nontrivial element of $\operatorname{Rep} D\left(\mathbb{S}_{n}\right)_{p t}$ corresponds to the pair $(e, \mathrm{Sg})$. We have

and

$$
s_{(e, \mathrm{Sg}),(e, \mathrm{Sg})}=\frac{\left|\mathbb{S}_{n}\right|}{\left|\mathbb{S}_{n}\right|^{2}} \sum_{g \in \mathbb{S}_{n}} \operatorname{Sg}(e) \operatorname{Sg}(e)=\frac{\left|\mathbb{S}_{n}\right|}{\left|\mathbb{S}_{n}\right|}=1,
$$

$$
\theta_{(e, \mathrm{Sg})}=\frac{\operatorname{Sg}(e)}{\operatorname{deg} \mathrm{Sg}}=1,
$$

where $\left(s_{X, Y}\right)_{X, Y \in \operatorname{Irr}\left(D\left(\mathbb{S}_{n}\right)\right)}$ and $\theta$ denote the $S$-matrix and the ribbon structure of $\operatorname{Rep} D\left(\mathbb{S}_{n}\right)$, respectively. See for instance [23, Section 3.1]. This shows that $\operatorname{Rep} D\left(\mathbb{S}_{n}\right)_{p t}$ is a Tannakian subcategory, as claimed.

Lemma 4.9. Let $n$ be an odd natural number. Then there is a central exact sequence of Hopf algebras

$$
k \longrightarrow k^{\mathbb{Z}_{2}} \longrightarrow J_{n} \stackrel{\pi}{\longrightarrow} K_{n} \longrightarrow k,
$$

where the map $\pi: J_{n} \rightarrow K_{n}$ is induced by the inclusion $\mathbb{A}_{n-1} \subseteq \mathbb{S}_{n-1}$.

Proof. The map $\pi$ is defined in the form $\pi=j \otimes \mathrm{id}: J_{n}=k^{\mathbb{S}_{n-1}} \# k C_{n} \rightarrow K_{n}=$ $k^{\mathbb{A}_{n-1}} \# k C_{n}$, where $j: k^{\mathbb{S}_{n-1}} \rightarrow k^{\mathbb{A}_{n-1}}$ is the canonical Hopf algebra map. Then $\pi$ is a surjective Hopf algebra map. 
Since the index of $K_{n}$ in $J_{n}$ is 2 , then $J_{n}^{\operatorname{co} \pi} \cong k \mathbb{Z}_{2} \cong k^{\mathbb{Z}_{2}}$ is a necessarily central Hopf subalgebra; see [26, Corollary 1.4.3].

Proposition 4.10. (i) $\operatorname{Rep} J_{n}$ is a $\mathbb{Z}_{2}$-extension of $\operatorname{Rep} K_{n}$, for all odd natural number $n \geq 1$.

(ii) $\operatorname{Rep} J_{n}$ is a $\mathbb{Z}_{2}$-equivariantization of a fusion category $\mathcal{D}$, for all even natural number $n \geq 4$.

Proof. (i) This is an immediate consequence of Lemma 4.9. That is, since the sequence (4.8) is a central exact sequence of Hopf algebras, then $\operatorname{Rep}\left(J_{n}\right)$ is an $\mathbb{Z}_{2^{-}}$ graded fusion category with trivial component $\left(\operatorname{Rep}\left(J_{n}\right)\right)_{0}=\operatorname{Rep}\left(K_{n}\right)$ [14, Theorem 3.8]. Therefore $\operatorname{Rep} J_{n}$ is a $\mathbb{Z}_{2}$-extension of $\operatorname{Rep} K_{n}$.

(ii) Suppose that $n \geq 4$ is even. We first claim that $\operatorname{Rep} J_{n}$ is not a $\mathbb{Z}_{2}$-extension of any fusion category. To see this, first note that it follows from [16, Lemma 3.4] that $G\left(J_{n}\right)=G\left(k^{\mathbb{S}_{n-1}}\right) \cong \mathbb{Z}_{2}$, for all $n \geq 2$. Suppose that $K$ is a central Hopf subalgebra of $J_{n}$ such that $K \cong k^{\mathbb{Z}_{2}}$, then $K$ must necessarily coincide with $k G\left(J_{n}\right)$. Observe that the action $\triangleleft: \mathbb{S}_{n-1} \times C_{n} \rightarrow \mathbb{S}_{n-1}$ gives rise to an action by algebra automorphism

$$
\rightarrow: C_{n} \times k^{\mathbb{S}_{n-1}} \rightarrow k^{\mathbb{S}_{n-1}} \text { such that } x \rightarrow e_{\sigma}=e_{\sigma \triangleleft x^{-1}},
$$

for all $x \in C_{n}$ and $\sigma \in \mathbb{S}_{n-1}$. If $\epsilon \neq \varphi \in G\left(J_{n}\right)=G\left(k^{\mathbb{S}_{n-1}}\right)$, we have $\varphi(\sigma)=\operatorname{Sg}(\sigma)$, for all $\sigma \in \mathbb{S}_{n-1}$ and $\varphi=\sum_{\sigma \in \mathbb{S}_{n-1}} \operatorname{Sg}(\sigma) e_{\sigma}$.

Then $G\left(J_{n}\right) \subseteq Z\left(J_{n}\right)$ if and only if $z \rightarrow \varphi=\varphi$, if and only if $\mathbb{A}_{n-1}$ is stable under the action $\triangleleft$ of $\langle z\rangle$. This contradicts the observation in Remark 4.6. Hence $\operatorname{Rep} J_{n}$ is not a $\mathbb{Z}_{2}$-extension of any fusion category, as claimed.

Let $\mathcal{E}=\operatorname{Rep} D\left(\mathbb{S}_{n}\right)_{p t}$. By Lemma 4.8, $\mathcal{E} \cong \operatorname{Rep} \mathbb{Z}_{2}$ is a Tannakian subcategory of $\operatorname{Rep} D\left(\mathbb{S}_{n}\right)$. Since Rep $J_{n}$ is not a $\mathbb{Z}_{2}$-extension of any fusion category, it follows from [12, Propositions 2.9 and 2.10] that Rep $J_{n}$ must be a $\mathbb{Z}_{2}$-equivariantization of a fusion category $\mathcal{D}$. We thus obtain (ii).

Lemma 4.11. Let $n \geq 5$ be an odd natural number and let $q$ be a prime number. Then the following hold:

(i) The category $\operatorname{Rep} J_{n}$ is not a $\mathbb{Z}_{q}$-equivariantization of any fusion category.

(ii) Suppose that $\operatorname{Rep} J_{n}$ is a $\mathbb{Z}_{q}$-extension of a fusion category $\tilde{\mathcal{D}}$. Then $q=2$ and $\tilde{\mathcal{D}}=\operatorname{Rep} K_{n}$.

(iii) The category $\operatorname{Rep} K_{n}$ is neither a $\mathbb{Z}_{q}$-equivariantization nor a $\mathbb{Z}_{q}$-extension of any fusion category.

Proof. Let $\mathcal{C}$ be one of the categories $\operatorname{Rep} J_{n}$ or $\operatorname{Rep} K_{n}$. If $\mathcal{C}$ is a $\mathbb{Z}_{q}$-extension of a fusion category $\tilde{\mathcal{D}}$, then the Drinfeld center $\mathcal{Z}(\mathcal{C})$ must contain a Tannakian subcategory equivalent to $\operatorname{Rep} \mathbb{Z}_{q}$ which maps to the trivial fusion subcategory Vect $\subseteq \mathcal{C}$ under the forgetful functor $U: \mathcal{Z}(\mathcal{C}) \rightarrow \mathcal{C}$. In this case, the category $\tilde{\mathcal{D}}$ is canonically determined by the corresponding Tannakian subcategory. Dually, if $\mathcal{C}$ is a $\mathbb{Z}_{q}$-equivariantization, then $\mathcal{Z}(\mathcal{C})$ must contain a Tannakian subcategory equivalent to $\operatorname{Rep} \mathbb{Z}_{q}$ which embeds into $\mathcal{C}$ under the forgetful functor. See [12, Propositions 2.9 and 2.10].

Since $n \geq 5$, then the group of invertible objects of the Drinfeld center of $\mathcal{C}$ coincides with $\mathbb{Z}_{2}$ if $\mathcal{C}=\operatorname{Rep} J_{n}$, and it is trivial if $\mathcal{C}=\operatorname{Rep} K_{n}$. Since $\operatorname{Rep} \mathbb{Z}_{q}$ is a pointed fusion category, we get (iii).

Suppose that $\mathcal{C}=\operatorname{Rep} J_{n}$. Then $\mathcal{C}$ is a $\mathbb{Z}_{2}$-extension of $\operatorname{Rep} K_{n}$. This implies that the pointed subcategory of $\mathcal{Z}(\mathcal{C})$ is a Tannakian subcategory which maps to the trivial subcategory of $\mathcal{C}$ under the forgetful functor. Hence, for every prime number $q, \mathcal{C}$ is not a $\mathbb{Z}_{q}$-equivariantization of any fusion category and we get (i). On the other hand, if it is $\mathbb{Z}_{q}$-extension, then $q=2$ and the corresponding Tannakian 
subcategory of $\mathcal{Z}(\mathcal{C})$ coincides with the pointed subcategory $\mathcal{Z}(\mathcal{C})_{p t}$. Thus we obtain (ii). This finishes the proof of the lemma.

Lemma 4.12. Let $p$ be an odd prime number. Then the group $G\left(J_{p}^{*}\right)$ is a semidirect product $\widehat{C_{p}} \rtimes\langle\sigma\rangle$ where $\sigma \in \mathbb{S}_{p-1}$ is a $(p-1)$-cycle and the action of $\langle\sigma\rangle$ on $\widehat{C_{p}}$ is induced by the action $\triangleleft^{\prime}: C_{p} \times \mathbb{S}_{p-1} \rightarrow C_{p}$. Moreover, the subgroup $G\left(K_{p}^{*}\right) \subseteq G\left(J_{p}^{*}\right)$ is the semidirect product $\widehat{C_{p}} \rtimes\left\langle\sigma^{2}\right\rangle$.

Proof. The subgroup $\mathbb{S}_{p-1}^{C_{p}}$ of invariants of $\mathbb{S}_{p-1}$ under the action $\triangleright^{\prime}$ of $C_{p}$ coincides with the subgroup of invariants under the action $\triangleleft$. It follows from [16, Corollary $5.2]$ that $\mathbb{S}_{p-1}^{C_{p}}$ is cyclic generated by a $(p-1)$ cycle $\sigma$, i.e. $\mathbb{S}_{p-1}^{C_{p}}=\langle\sigma\rangle$ and therefore $G\left(J_{p}^{*}\right) \cong \widehat{C_{p}} \rtimes\langle\sigma\rangle$. It follows from this that the invariant subgroup $\mathbb{A}_{p-1}^{C_{p}}$ is also cyclic generated by $\sigma^{2}$. This implies the lemma, in view of Remark 4.4.

Proposition 4.13. Let $p$ be an odd prime number. Then the Hopf algebras $J_{p}, K_{p}$ satisfy the following properties:

(i) $\operatorname{cd}\left(J_{p}\right)=\operatorname{cd}\left(K_{p}\right)=\{1, p\}$.

(ii) The groups $G\left(J_{p}^{*}\right)$ and $G\left(K_{p}^{*}\right)$ have trivial centers.

Proof. Part (i) follows from the description of irreducible representations of crossed products in [20].

We next show (ii). Recall that $C_{p}=\langle z\rangle$, where $z=(12 \ldots p)$ and the actions $\triangleleft$, $\triangleright$ are determined by the relation $s c=(s \triangleright c)(s \triangleleft c)$ in $\mathbb{S}_{p}$. So that the actions $\triangleleft^{\prime}$, $\triangleright^{\prime}$ are determined by $c s=\left(c \triangleright^{\prime} s\right)\left(c \triangleleft^{\prime} s\right)$ in $\mathbb{S}_{p}$, for all $s \in \mathbb{S}_{p-1}, c \in C_{p}$.

It follows from the proof of [16, Lemma 3.2] that $z \triangleleft^{\prime} a_{i}=z^{i}$, for all $i=$ $1, \ldots, p-1$, where $a_{i}=(p-1, p-i)$. In addition, the stabilizer of $z$ under the action $\triangleleft^{\prime}$ coincides with the subgroup $F_{z}=\left\{a \in \mathbb{S}_{p-1}: a(p-1)=p-1\right\} \cong \mathbb{S}_{p-2}$. These imply that, for all $i=1, \ldots, p-1$, the stabilizer of $z^{i}$ coincides with the subgroup $F_{z^{i}}=\left\{a \in \mathbb{S}_{p-1}: a(p-i)=p-i\right\}$. In particular, $C_{p}$ has no nontrivial fixed points under the action $\triangleleft^{\prime}$.

On the other hand, the nontrivial powers of the $(p-1)$-cycle $\sigma \in \mathbb{S}_{p-1}$ have no fixed point in $\{1, \ldots, p-1\}$. Hence no nontrivial power of $\sigma$ acts trivially on $C_{p}$ under the action $\triangleleft^{\prime}$.

By Lemma 4.12, $G\left(J_{p}^{*}\right)=\widehat{C_{p}} \rtimes\langle\sigma\rangle$ is a semidirect product with respect to the action induced by $\triangleleft^{\prime}$, where $\sigma$ is a $(p-1)$-cycle in $\mathbb{S}_{p-1}$. Then the center of $G\left(J_{p}^{*}\right)$ consists of all pairs $(e, x)$, where $x \in\langle\sigma\rangle$ acts trivially on $C_{p}$ under the action $\triangleleft^{\prime}$.

Similarly, $G\left(K_{p}^{*}\right)=\widehat{C_{p}} \rtimes\left\langle\sigma^{2}\right\rangle$ is a semidirect product with respect to the action induced by $\triangleleft^{\prime}$ and the center of $G\left(K_{p}^{*}\right)$ consists of all pairs $(e, x)$, where $x \in\left\langle\sigma^{2}\right\rangle$ acts trivially on $C_{p}$ under the action $\triangleleft^{\prime}$.

Thus we obtain that the centers of the groups $G\left(J_{p}^{*}\right)$ and $G\left(K_{p}^{*}\right)$ are both trivial.

Theorem 4.14. Let $\tilde{\mathcal{C}}$ be a fusion category. Suppose that $\tilde{\mathcal{C}}$ is Grothendieck equivalent to one of the categories $\operatorname{Rep} J_{p}$ or $\operatorname{Rep} K_{p}$. Then $\tilde{\mathcal{C}}$ is not solvable.

Proof. Suppose on the contrary that $\tilde{\mathcal{C}}$ is solvable and Grothendieck equivalent to $\mathcal{C}$, where $\mathcal{C}=\operatorname{Rep} J_{p}$ or $\operatorname{Rep} K_{p}$. It follows from Proposition 3.2 that $G(\tilde{\mathcal{C}}) \cong G(\mathcal{C})$. By Proposition 4.13, the groups of invertible objects of Rep $J_{p}$ and $\operatorname{Rep} K_{p}$ have trivial center. Then the center of $G(\tilde{\mathcal{C}})$ is trivial as well. It follows from Lemma 2.3 that, for every prime number $q$, the category $\tilde{\mathcal{C}}$ is not a $\mathbb{Z}_{q}$-equivariantization of any fusion category. Therefore $\tilde{\mathcal{C}}$ must be a $\mathbb{Z}_{q}$-extension of a fusion subcategory $\tilde{\mathcal{D}}$, and $\tilde{\mathcal{D}}$ is also a solvable fusion category. Hence $\mathcal{C}$ is a $\mathbb{Z}_{q}$-extension of a fusion subcategory $\mathcal{D}$ such that $\tilde{\mathcal{D}}$ is Grothendieck equivalent to $\mathcal{D}$. It follows from Proposition 4.10 
and Lemma 4.11 that $\mathcal{C}=\operatorname{Rep} J_{p}, q=2$ and $\mathcal{D}=\operatorname{Rep} K_{p}$. Applying the same argument to the solvable fusion category $\tilde{\mathcal{D}}$ we get a contradiction. This shows that $\tilde{\mathcal{C}}$ cannot be solvable and finishes the proof of the theorem.

4.3. Fusion rules of $\operatorname{Rep} K_{5}$. In this subsection we determine explicitly the fusion rules of the category $\operatorname{Rep} K_{p}$ in the case $p=5$. It follows from 20] that simple objects of the category Rep $K_{5}$ are parameterized by pairs $(s, \rho)$, where $s$ runs over a set of representatives of the orbits of $C_{5}$ on $\mathbb{A}_{4}$ and $\rho$ is an irreducible representation of the stabilizer $F_{s} \subseteq C_{5}$. The dimension of the simple object $S_{s, \rho}$ corresponding to the pair $(s, \rho)$ is given by the formula $\operatorname{dim} S_{s, \rho}=\left[C_{5}: F_{s}\right]$.

The $C_{5}$-action on $\mathbb{S}_{4}$ is explicitly determined in [16, Table 1]. We have in this case that there are 10 fixed points and the remaining 2 orbits consist of 5 distinct elements each. Furthermore, there are exactly 4 distinct fixed points $\sigma$ such that $\sigma=\sigma^{-1}$ and both nontrivial orbits contain elements of order 2. In view of [16, Theorem 4.8], Rep $K_{5}$ has 5 invertible objects of order 2 and the 5-dimensional simple objects are self-dual.

Let us denote by $Y, Y^{\prime}$ the simple objects corresponding to the nontrivial orbits $\mathcal{O}, \mathcal{O}^{\prime}$, respectively. By [16, Table 1], we have

$$
\begin{aligned}
& \mathcal{O}=\{(123),(243),(132),(13)(24),(234)\}, \\
& \mathcal{O}^{\prime}=\{(124),(143),(134),(12)(34),(142)\} .
\end{aligned}
$$

By Lemma 4.12 the group $G\left(\operatorname{Rep} K_{5}\right)$ is isomorphic to the dihedral group $D_{5}$ of order 10. The unique subgroup $R$ of order 5 of $G\left(\operatorname{Rep} K_{5}\right)$ coincides therefore with the stabilizer of $Y$ and $Y^{\prime}$ under left (or right) multiplication. Since every element $s$ outside of $R$ is of order 2 , then $s \otimes Y \cong Y \otimes s \cong Y^{\prime}$. So that we have a decomposition

$$
Y \otimes Y^{*} \cong Y \otimes Y \cong \bigoplus_{r \in R} r \oplus a Y \oplus b Y^{\prime}
$$

where $a, b \geq 0$ and $a+b=4$. Letting $F: \operatorname{Rep} K_{5} \rightarrow \mathcal{C}\left(\mathbb{A}_{4}\right)=\operatorname{Rep} k^{\mathbb{A}_{4}}$ denote the restriction functor, we obtain

$$
\begin{aligned}
& F(Y)=V_{(123)} \oplus V_{(243)} \oplus V_{(132)} \oplus V_{(13)(24)} \oplus V_{(234),}, \\
& F\left(Y^{\prime}\right)=V_{(124)} \oplus V_{(143)} \oplus V_{(134)} \oplus V_{(12)(34)} \oplus V_{(142)},
\end{aligned}
$$

where, for each $s \in \mathbb{A}_{4}, V_{s}$ denotes the one-dimensional simple $k^{\mathbb{A}_{4}}$-module corresponding to $s$. Comparing these relations with (4.9), we find that $a=b=2$. Hence the fusion rules of $\operatorname{Rep} K_{5}$ are determined by the condition $G=G\left(\operatorname{Rep} K_{5}\right) \cong D_{5}$, $g \otimes Y=Y \otimes g=Y^{\prime}$, for every element or order 2 of $G$, and

$$
Y \otimes Y \cong \bigoplus_{r \in R} r \oplus 2 Y \oplus 2 Y^{\prime} \cong Y^{\prime} \otimes Y^{\prime}
$$

where $R$ is the unique subgroup of order 5 of $G$.

4.4. The dual Hopf algebras $J_{n}^{*}, K_{n}^{*}$. Let $n \geq 2$ be a natural number and let $H_{n}=J_{n}^{*}$. Recall that there is a split exact sequence of Hopf algebras

$$
k \longrightarrow k^{C_{n}} \longrightarrow H_{n} \longrightarrow k \mathbb{S}_{n-1} \longrightarrow k \text {. }
$$

Suppose that $n$ is odd. Let $L_{n}=K_{n}^{*}$, so that there is a split exact sequence of Hopf algebras

$$
k \longrightarrow k^{C_{n}} \longrightarrow L_{n} \longrightarrow k \mathbb{A}_{n-1} \longrightarrow k .
$$

Moreover, by Lemma 4.9 there is a cocentral exact sequence

$$
k \longrightarrow L_{n} \longrightarrow H_{n} \longrightarrow k \mathbb{Z}_{2} \longrightarrow k \text {. }
$$


Remark 4.15. Suppose $n \geq 5$. Since $D\left(H_{n}\right) \cong D\left(J_{n}\right)$, then $G\left(\operatorname{Rep} D\left(H_{n}\right)\right) \cong \mathbb{Z}_{2}$. Let $q$ be a prime number. If the category $\operatorname{Rep} H_{n}$ is a $\mathbb{Z}_{q^{-}}$extension or a $\mathbb{Z}_{q^{-}}$ equivariantization of a fusion category, then $q=2$.

Suppose $n$ is odd. In view of [27, Proposition 3.5], Rep $H_{n}$ is a $\mathbb{Z}_{2}$-equivariantization of Rep $L_{n}$. As in the proof of Lemma4.11, we obtain that if $n \geq 5$, the category $\operatorname{Rep} H_{n}$ is not a $\mathbb{Z}_{q^{-}}$-extension of any fusion category. Similarly, $\operatorname{Rep} L_{n}$ is not a $\mathbb{Z}_{q^{-}}$ extension or a $\mathbb{Z}_{q}$-equivariantization of any fusion category.

Lemma 4.16. Let $n \geq 5$ be a natural number. Then the following hold.

(i) $G\left(\operatorname{Rep} H_{n}\right) \cong \mathbb{Z}_{2}$.

(ii) $\operatorname{Rep}\left(H_{5}\right)$ is of type $(1,2 ; 2,1 ; 3,2 ; 4,2 ; 8,1)$ and $H_{n}$ has no irreducible representation of dimension 2, for all $n>5$.

Assume in addition that $n$ is odd. Then

(iii) $G\left(\operatorname{Rep} L_{n}\right)=1$, if $n>5$.

(iv) Rep $L_{5}$ is of type $(1,3 ; 3,1 ; 4,3)$ and $L_{n}$ has no irreducible representation of dimension 2.

Proof. Consider the exact sequences (4.10), (4.11). The respective invariant subgroups $C_{n}^{\mathbb{S}_{n-1}}$ and $C_{n}^{\mathbb{A}_{n-1}}$ are both trivial. Parts (i) and (iii) follow from Lemma 4.3

Since $H_{n}$ is a split abelian extension of $k^{C_{n}}$ by $k \mathbb{S}_{n-1}$ and the action of $\mathbb{S}_{n-1}$ has two orbits $\{e\}$ and $\left\{z, \ldots, z^{n-1}\right\}$, then the simple $H_{n}$-modules are classified by pairs $(t, \rho)$, where either $t=e$ and $\rho$ is an irreducible representation of $F_{e}=\mathbb{S}_{n-1}$, or $t=z$ and $\rho$ is an irreducible representation of $F_{z}=\left\{a \in \mathbb{S}_{n-1}: a(n-1)=n-1\right\} \cong \mathbb{S}_{n-2}$. See [16, Lemma 3.2]. If $S_{t, \rho}$ is the simple module corresponding to the pair $(t, \rho)$, we have in addition $\operatorname{dim} S_{e, \rho}=\operatorname{dim} \rho$, and $\operatorname{dim} S_{z, \rho}=\left[\mathbb{A}_{n-1}: F_{z}\right] \operatorname{dim} \rho=(n-1) \operatorname{dim} \rho$. This implies the statement for $H_{5}$ in part (ii).

Suppose that $n>5$. Then $\operatorname{dim} S_{z, \rho}>2$, for all $\rho$. As observed in Remark 3.6, the symmetric group $\mathbb{S}_{n-1}$ has no irredubible representation of degree 2 . Therefore we also get that $\operatorname{dim} S_{e, \rho} \neq 2$, for all $\rho$. In conclusion $H_{n}$ has no irreducible representation of dimension 2 , and we obtain (ii).

Suppose that $n$ is odd. Similarly, $L_{n}$ is a split abelian extension of $k^{C_{n}}$ by $k \mathbb{A}_{n-1}$ and the action of $\mathbb{A}_{n-1}$ has two orbits $\{e\}$ and $\left\{z, \ldots, z^{n-1}\right\}$. Hence the simple $L_{n}$-modules are classified by pairs $(t, \rho)$, where either $t=e$ and $\rho$ is an irreducible representation of $F_{e} \cap \mathbb{A}_{n-1}=\mathbb{A}_{n-1}$, or $t=z$ and $\rho$ is an irreducible representation of $F_{z} \cap \mathbb{A}_{n-1} \cong \mathbb{A}_{n-2}$. This implies that Rep $L_{5}$ is of the prescribed type. As before, $\operatorname{dim} S_{z, \rho}>2$, for all $\rho$, and since the alternating group $\mathbb{A}_{n-1}$ has no irredubible representation of degree 2 , then also $\operatorname{dim} S_{e, \rho} \neq 2$, for all $\rho$. So that $L_{n}$ has no irreducible representation of dimension 2. This proves part (iv) and finishes the proof of the lemma.

Lemma 4.17. Let $\mathcal{C}$ be a fusion category of type $(1,3 ; 3,1 ; 4,3)$. Then $\mathcal{C}$ is not solvable.

Proof. The assumption on the type of $\mathcal{C}$ implies that the simple objects of dimensions 1 and 3 generate a fusion subcategory $\mathcal{B}$ of $\mathcal{C}$ of type $(1,3 ; 3,1)$ and moreover, every fusion subcategory of $\mathcal{C}$ is contained in $\mathcal{B}$.

Suppose first that $\mathcal{C}$ is a $\mathbb{Z}_{q}$-extension of a fusion subcategory $\mathcal{C}_{0}$, for some prime number $q$. Then necessarily $\mathcal{C}_{0}=\mathcal{B}$ and $q=5$. Hence we have a $\mathbb{Z}_{5}$-faithful grading $\mathcal{C}=\mathcal{C}_{0} \oplus \mathcal{C}_{1} \oplus \cdots \oplus \mathcal{C}_{4}$, with trivial component $\mathcal{C}_{0}=\mathcal{B}$. But $\mathcal{C}$ has only 3 classes of simple objects outside of $\mathcal{B}$, entailing that such a decomposition is impossible.

Therefore $\mathcal{C}$ must be a $\mathbb{Z}_{q}$-equivariantization of a fusion category $\mathcal{D}$, for some prime number $q$, where $\mathcal{D}$ is also a solvable fusion category. Thus $q=3$ and $\mathcal{C} \cong \mathcal{D}^{\mathbb{Z}_{3}}$. The description of simple objects of $\mathcal{D}^{\mathbb{Z}_{3}}$ together with the assumption 
on the type of $\mathcal{C}$ imply that $\mathcal{D}$ must be of type $(1,4 ; 4,1)$; c.f. Formula (2.2). Moreover, the action (by group automorphisms) of $\mathbb{Z}_{3}$ on the set of nontrivial invertible objects of $\mathcal{D}$ must be transitive, hence $G(\mathcal{D}) \cong \mathbb{Z}_{2} \times \mathbb{Z}_{2}$.

On the other hand, letting $X$ be the unique noninvertible simple object of $\mathcal{D}$, we must have

$$
X^{\otimes 2} \cong \oplus_{Y \in G(\mathcal{D})} Y \oplus 3 X .
$$

This means that $\mathcal{D}$ is a near-group fusion category of type $(G, 3)$, where $G=G(\mathcal{D})$. Then it follows from [31, Theorem 1.2] that the group $G(\mathcal{D})$ is cyclic, which leads to a contradiction. Therefore $\mathcal{C}$ cannot be solvable. This finishes the proof of the lemma.

Theorem 4.18. Let $\mathcal{C}$ be a fusion category and let $n \geq 5$ be a natural number. Then we have:

(i) If $\mathcal{C}$ is Grothendieck equivalent to $\operatorname{Rep} H_{n}$, then $\mathcal{C}$ is not solvable.

(ii) Suppose that $n$ is odd. If $\mathcal{C}$ is Grothendieck equivalent to $\operatorname{Rep} L_{n}$, then $\mathcal{C}$ is not solvable.

Proof. To show part (i), suppose first that $n>5$. By Lemma 4.16 $G\left(\operatorname{Rep} H_{n}\right) \cong \mathbb{Z}_{2}$ and Rep $H_{n}$ has no simple objects of dimension 2. The claim follows in this case from Proposition 3.5 .

Consider the case $n=5$. Suppose on the contrary that $\mathcal{C}$ is Grothendieck equivalent to $\operatorname{Rep} H_{5}$ and $\mathcal{C}$ is solvable. Then $\mathcal{C}$ is of type $(1,2 ; 2,1 ; 3,2 ; 4,2 ; 8,1)$ and, for any prime $q, \mathcal{C}$ is not a $\mathbb{Z}_{q}$-extension of any fusion subcategory, in view of Remark 4.15. Therefore $\mathcal{C}$ must be a $\mathbb{Z}_{2}$-equivariantization of a solvable fusion category $\mathcal{D}$ of dimension 60 . The description of the simple objects of $\mathcal{D}^{\mathbb{Z}_{2}}$ together with the assumption on the type of $\mathcal{C}$ imply that $\mathcal{D}$ must be of type $(1,3 ; 3,1 ; 4,3)$. Lemma 4.17 implies that $\mathcal{D}$ is not solvable, which is a contradiction. Thus we get (i).

Let us show (ii). If $n=5$, the result follows from Lemma 4.17. Suppose next that $n>5$. Since a solvable fusion category contains nontrivial invertible objects, then part (ii) follows from Lemma 4.16 (iii).

4.5. Further examples associated to the symmetric group. Let $n \geq 2$ be a natural number. Consider the matched pair $\left(\mathbb{A}_{n}, C_{2}\right)$, where $C_{2}=\langle(12)\rangle \subseteq \mathbb{S}_{n}$, the action $\triangleleft: \mathbb{A}_{n} \times C_{2} \rightarrow \mathbb{A}_{n}$ is given by conjugation in $\mathbb{S}_{n}$ and the action $\triangleright$ : $\mathbb{A}_{n} \times C_{2} \rightarrow C_{2}$ is trivial. The associated group $\mathbb{A}_{n} \bowtie C_{2}$ is isomorphic to the symmetric group $\mathbb{S}_{n}$.

Let $B_{n}=k^{\mathbb{A}_{n}} \# k C_{2}$ be the split abelian extension associated to this matched pair. We have $\operatorname{cd}\left(B_{n}\right)=\{1,2\}$. The fusion category $\operatorname{Rep} B_{n}$ is Morita equivalent to $\mathbb{S}_{n}$ and therefore it is not solvable if $n \geq 5$.

Remark 4.19. Since the action $\triangleright$ is the trivial one and $C_{2} \cong \mathbb{Z}_{2}$, there is a cocentral exact sequence

$$
k \longrightarrow k^{\mathbb{A}_{n}} \longrightarrow B_{n} \longrightarrow k \mathbb{Z}_{2} \longrightarrow k .
$$

In view of [27, Proposition 3.5] $\operatorname{Rep} B_{n}$ is a $\mathbb{Z}_{2}$-equivariantization of $\operatorname{Rep} k_{n}^{\mathbb{A}}=$ $\mathcal{C}\left(\mathbb{A}_{n}\right)$.

Moreover, since $\operatorname{Rep} B_{n}$ is Morita equivalent to $\mathbb{S}_{n}$ and the group of invertible objects of $\mathcal{Z}\left(\operatorname{Rep} \mathbb{S}_{n}\right)$ is cyclic of order 2 , then for all prime number $q$, $\operatorname{Rep} B_{n}$ is not a $\mathbb{Z}_{q}$-extension of any fusion category and if it is a $\mathbb{Z}_{q}$-equivariantization, then $q=2$ (compare with Proposition 4.10). In particular, not being a $\mathbb{Z}_{q}$-extention, $B_{n}$ has no nontrivial central group-like elements; that is, $Z\left(B_{n}\right) \cap G\left(B_{n}\right)=\{1\}$.

Our first statement concerns the dual Hopf algebra $B_{n}^{*}$. 
Theorem 4.20. Let $\mathcal{C}$ be a fusion category. Suppose that $\mathcal{C}$ is Grothendieck equivalent to $\operatorname{Rep} B_{n}^{*}, n \geq 5$. Then $\mathcal{C}$ is not solvable.

Proof. The dual Hopf algebra $B_{n}^{*}$ fits into a central exact sequence

$$
k \longrightarrow k^{\mathbb{Z}_{2}} \longrightarrow B_{n} \longrightarrow k \mathbb{A}_{n} \longrightarrow k \text {. }
$$

Therefore Rep $B_{n}^{*}$ is a $\mathbb{Z}_{2}$-extension of Rep $\mathbb{A}_{n}$. Hence $\mathcal{C}$ is a $\mathbb{Z}_{2}$-extension of a fusion category $\mathcal{D}$, which is Grothendieck equivalent to $\operatorname{Rep} \mathbb{A}_{n}$.

Suppose on the contrary that $\mathcal{C}$ is solvable. Then so is $\mathcal{D}$ and therefore $\mathcal{D}_{p t} \neq$ Vect. This implies that $\mathbb{A}_{n}$ has nontrivial one-dimensional representations, which is a contradiction. Then $\mathcal{C}$ cannot be solvable, as claimed.

Lemma 4.21. The group $G\left(\operatorname{Rep} B_{n}\right)$ of invertible objects of the category $\operatorname{Rep} B_{n}$ is isomorphic to the direct product $\widehat{C_{2}} \times C_{\mathbb{A}_{n}}(12)$, where $C_{\mathbb{A}_{n}}(12)$ denotes the centralizer in $\mathbb{A}_{n}$ of the transposition (12).

Proof. There is a group isomorphism $G\left(\operatorname{Rep} B_{n}\right) \cong G\left(B_{n}^{*}\right)$. On the other hand, $B_{n}^{*}$ is a split abelian extension $B_{n}^{*} \cong k^{C_{2}} \# k \mathbb{A}_{n}$, associated to the adjoint action $\triangleright^{\prime}: C_{2} \times \mathbb{A}_{n} \rightarrow \mathbb{A}_{n}$ and the trivial action $\triangleleft^{\prime}: C_{2} \times \mathbb{A}_{n} \rightarrow C_{2}$. The result follows from Lemma 4.3

Theorem 4.22. Suppose $n \geq 5$. Let $\tilde{\mathcal{C}}$ be a fusion category Grothendieck equivalent to $\operatorname{Rep} B_{n}$. Then $\tilde{\mathcal{C}}$ is not solvable.

Proof. Suppose first that $n \geq 7$. By Lemma 4.21. $G\left(\operatorname{Rep} B_{n}\right) \cong \widehat{C_{2}} \times C_{\mathbb{A}_{n}}(12)$. Note that $C_{\mathbb{A}_{n}}(12)$ contains the subgroup $\left\{\sigma \in \mathbb{A}_{n}: \sigma(1)=1, \sigma(2)=2\right\} \cong \mathbb{A}_{n-2}$. Since $n \geq 7$, the group $\mathbb{A}_{n-2}$ is not solvable. Then $G(\tilde{\mathcal{C}})$ is not solvable neither and then $\tilde{\mathcal{C}}$ is not solvable.

It remains to consider the cases $n=5$ and 6 . It follows from Lemma 4.21 that $G\left(\operatorname{Rep} B_{5}\right) \cong \widehat{C_{2}} \times \mathbb{S}_{3}$ is non-abelian of order 12 . Hence Rep $B_{5}$ is of type $(1,12 ; 2,27)$. Similarly, $G\left(\operatorname{Rep} B_{6}\right) \cong \widehat{C_{2}} \times \mathbb{S}_{4}$ is non-abelian of order 48 and $\operatorname{Rep} B_{6}$ is of type $(1,48 ; 2,168)$.

Suppose that there exist a solvable fusion category $\tilde{\mathcal{C}}$ which is Grothendieck equivalent to $\mathcal{C}$, where $\mathcal{C}=\operatorname{Rep} B_{5}$ or $\operatorname{Rep} B_{6}$. By Proposition $3.2, G(\tilde{\mathcal{C}}) \cong G(\mathcal{C})$. Since, for every prime number $q, \mathcal{C}$ is not a $\mathbb{Z}_{q}$-extension of any fusion category, we have that $\tilde{\mathcal{C}}$ must be a $\mathbb{Z}_{q}$-equivariantization of a fusion subcategory $\mathcal{D}$, and $\mathcal{D}$ is also a solvable fusion category. Moreover, $q=2$ because $\mathbb{Z}_{q} \subseteq Z(G(\tilde{\mathcal{C}}))$ and $\mathrm{FP} \operatorname{dim} \mathcal{D}=60$ or FPdim $\mathcal{D}=360$, respectively. Then there is an exact sequence of fusion categories

$$
\operatorname{Rep} \mathbb{Z}_{2} \rightarrow \tilde{\mathcal{C}} \rightarrow \mathcal{D} .
$$

Since $\operatorname{cd}(\tilde{\mathcal{C}})=\{1,2\}$, it follows that $\operatorname{cd}(\mathcal{D})=\{1,2\}$. The previous exact sequence induces an exact sequence of groups

$$
1 \rightarrow \widehat{\mathbb{Z}}_{2} \rightarrow G(\tilde{\mathcal{C}}) \rightarrow G_{0}(\mathcal{D}) \rightarrow 1
$$

where $\widehat{\mathbb{Z}}_{2}$ denotes the group of invertible characters of $\mathbb{Z}_{2}$ and $G_{0}(\mathcal{D})$ is the subgroup of $G(\mathcal{D})$ consisting of isomorphism classes of invertible objects which are $\mathbb{Z}_{2}$-equivariant. See [5, Remark 3.1]. As $\mathbb{Z}_{2}$ is a cyclic group, we have that $G_{0}(\mathcal{D})$ coincides with the subgroup of fixed points of the induced action of $\mathbb{Z}_{2}$ on the group of invertible objects of $\mathcal{D}$.

Observe that, since $\mathcal{C}$ is also a $\mathbb{Z}_{2}$-equivariantization of $\mathcal{C}_{\mathbb{Z}_{2}} \cong \mathcal{C}\left(\mathbb{A}_{5}\right)$ or $\mathcal{C}\left(\mathbb{A}_{6}\right)$, respectively, then the group $G(\mathcal{C}) \cong G(\tilde{\mathcal{C}})$ also fits into an exact sequence

$$
1 \rightarrow \widehat{\mathbb{Z}}_{2} \rightarrow G(\mathcal{C}) \rightarrow G_{0}\left(\mathcal{C}_{\mathbb{Z}_{2}}\right) \rightarrow 1 \text {. }
$$


In this case, the subgroup $G_{0}\left(\mathcal{C}_{\mathbb{Z}_{2}}\right)$ is isomorphic to $C_{\mathbb{A}_{5}}(12)$ or $C_{\mathbb{A}_{6}}(12)$, respectively. In addition, the group $G(\mathcal{C})$ contains a unique normal subgroup of order 2 . Therefore, $G_{0}(\mathcal{D}) \cong G_{0}\left(\mathcal{C}_{\mathbb{Z}_{2}}\right)$ is a non-abelian subgroup of $G(\mathcal{D})$.

Suppose first that $n=5$. In this case $\mathcal{C}=\operatorname{Rep} B_{5} \cong \mathcal{C}\left(\mathbb{A}_{5}\right)^{\mathbb{Z}_{2}}$. In particular, $G_{0}(\mathcal{D})$ is a subgroup of order 6 of $G(\mathcal{D})$. A counting argument shows that $G(\mathcal{D})$ can be of type $(1,12 ; 2,12)$ or else $\mathcal{D}$ is pointed. Suppose that $\mathcal{D}$ is of type $(1,12 ; 2,12)$. Then $\mathcal{D}_{p t}^{\mathbb{Z}_{2}} \subseteq \tilde{\mathcal{C}}$ is a fusion subcategory of dimension 24 and type $(1,12 ; 2,3)$, containing the central subcategory $\operatorname{Rep} \mathbb{Z}_{2}$. Therefore $\mathcal{C}$ has a fusion subcategory $\mathcal{B}$ of type $(1,12 ; 2,3)$ containing the central subcategory $\operatorname{Rep} \mathbb{Z}_{2}$. (In fact, as observed before, $G(\mathcal{C})$ contains a unique normal subgroup of order 2; see Lemma 2.3.)

Consider the de-equivariantization $\mathcal{B}_{\mathbb{Z}_{2}} \subseteq \mathcal{C}\left(\mathbb{A}_{5}\right)$. We have that $\operatorname{dim} \mathcal{B}_{\mathbb{Z}_{2}}=12$ and thus $\mathcal{B}_{\mathbb{Z}_{2}}=\mathcal{C}(H)$, where $H$ is a $\left(\mathbb{Z}_{2}\right.$-stable) subgroup of $\mathbb{A}_{5}$ of order 12 . Since $G\left(\operatorname{Rep} B_{5}\right) \subseteq \mathcal{B}$, then the subgroup $H$ contains the invariant sugroup $\mathbb{A}_{5}^{\mathbb{Z}_{2}}=$ $C_{\mathbb{A}_{5}}(12) \cong \mathbb{S}_{3}$. On the other hand, every subgroup of order 12 of $\mathbb{A}_{5}$ is isomorphic to $\mathbb{A}_{4}$, then $H \cong \mathbb{A}_{4}$. This leads to a contradiction, because $\mathbb{A}_{4}$ has no subgroup of order 6 . This proves that $\mathcal{D}$ cannot be of type $(1,12 ; 2,12)$.

Therefore $\mathcal{D}$ must be a pointed fusion category. In this case $\mathcal{D}=\mathcal{C}(\Gamma, \omega)$, where $\omega: \Gamma \times \Gamma \times \Gamma \rightarrow k^{*}$ is a 3-cocyle and $\Gamma$ is a solvable non-abelian subgroup of order 60 . In addition $\mathbb{Z}_{2}$ acts on $\Gamma$ by group automorphisms and $\Gamma^{\mathbb{Z}_{2}} \cong \mathbb{S}_{3}$. Since $\Gamma \neq \mathbb{A}_{5}$, $\Gamma$ can be isomorphic to $\mathbb{A}_{4} \times \mathbb{Z}_{5}, \mathbb{Z}_{15} \rtimes \mathbb{Z}_{4}$ or $\mathbb{Z}_{15} \rtimes\left(\mathbb{Z}_{2} \times \mathbb{Z}_{2}\right)$.

If $\Gamma \cong \mathbb{A}_{4} \times \mathbb{Z}_{5}$, the action of $\mathbb{Z}_{2}$ must fix $\mathbb{A}_{4}$ and $\mathbb{Z}_{5}$. Since $\left|\Gamma^{\mathbb{Z}_{2}}\right|=6$ then $\Gamma^{\mathbb{Z}_{2}} \subseteq \mathbb{A}_{4}$, and we reach a contradiction. Therefore $\Gamma \cong \mathbb{Z}_{15} \rtimes \mathbb{Z}_{4}$ or $\mathbb{Z}_{15} \rtimes\left(\mathbb{Z}_{2} \times \mathbb{Z}_{2}\right)$. In this case $\Gamma$ has a unique subgroup $L$ of order 15 , and then $L$ is $\mathbb{Z}_{2}$-stable and $\mathcal{C}(L)^{\mathbb{Z}_{2}}$ is a fusion subcategory of $\tilde{\mathcal{C}}$ of dimension 30 . This implies that $\mathcal{C}$ has a fusion subcategory of dimension 30. Such fusion subcategory must correspond to a quotient Hopf algebra of $B_{5}$ of dimension 30, which is a contradiction, because $Z\left(B_{5}\right) \cap G\left(B_{5}\right)=\{1\}$. See [26, Corollary 1.4.3]. Thus $\mathcal{D}$ cannot be pointed. This proves that if $\tilde{\mathcal{C}}$ is Grothendieck equivalent to $\operatorname{Rep} B_{5}$ then $\tilde{\mathcal{C}}$ is not solvable.

Finally, let us consider the case $n=6$. In this case we have $\mathcal{C}:=\operatorname{Rep} B_{6} \cong$ $\mathcal{C}\left(\mathbb{A}_{6}\right)^{\mathbb{Z}_{2}}$. On the other hand, $G_{0}(\mathcal{D})$ is a subgroup of order 24 of $G(\mathcal{D})$. As before, one can see that $\mathcal{D}$ must be of type $(1,24 ; 2,84),(1,72 ; 2,72),(1,120 ; 2,60)$, or else $\mathcal{D}$ is pointed.

Suppose that $\mathcal{D}$ is of type $(1,72 ; 2,72)$ or $(1,120 ; 2,60)$. In these cases $\mathcal{D}_{p t}^{\mathbb{Z}_{2}} \subseteq \tilde{\mathcal{C}}$ is a fusion subcategory of dimension 144 or 240 , respectively, containing the central subcategory $\operatorname{Rep} \mathbb{Z}_{2}$. Therefore $\mathcal{C}$ has a fusion subcategory $\mathcal{B}$ of dimension 144 or 240 , respectively, containing the central subcategory $\operatorname{Rep} \mathbb{Z}_{2}$. The deequivariantization $\mathcal{B}_{\mathbb{Z}_{2}} \subseteq \mathcal{C}\left(\mathbb{A}_{6}\right)$ is of dimension $\operatorname{dim} \mathcal{B}_{\mathbb{Z}_{2}}=72$ or 120 , respectively. Then $\mathcal{B}_{\mathbb{Z}_{2}}=\mathcal{C}(H)$, where $H$ is a $\mathbb{Z}_{2}$-stable subgroup of $\mathbb{A}_{6}$ of order 72 or 120 , respectively. Since $\mathbb{A}_{6}$ has no subgroups of order 72 or 120 , it follows that these types are not possible for $\mathcal{D}$.

Suppose next that $\mathcal{D}$ is of type $(1,24 ; 2,84)$. It follows from the description of the simple objects of $\mathcal{D}^{G}$ and the fact that $\operatorname{cd}(\mathcal{D})=\operatorname{cd}(\mathcal{C})=\{1,2\}$, that $\mathbb{Z}_{2}$ acts trivially on the set $\operatorname{Irr}(\mathcal{D})$; see (2.2). In particular, $G(\mathcal{D})=G_{0}(\mathcal{D}) \cong C_{\mathbb{A}_{6}}(12) \cong \mathbb{S}_{4}$.

Since $\mathcal{D}$ is solvable, then it is a $\mathbb{Z}_{p}$-extension or a $\mathbb{Z}_{p}$-equivariantization, where $p$ is a prime number that divides the dimension of $\mathcal{D}$, which is 360 . If $\mathcal{D}$ were a $\mathbb{Z}_{p}$-equivariantization then, by Lemma 2.3 $\mathbb{Z}_{p} \subseteq Z(G(\mathcal{D}))$, which is a contradiction because $G(\mathcal{D}) \cong \mathbb{S}_{4}$. Therefore $\mathcal{D}$ must be a $\mathbb{Z}_{p}$-extension of a fusion subcategory $\mathcal{D}_{e}$. The fusion subcategory $\mathcal{D}_{e}$ is of dimension 72,120 or 180 . Furthermore, $\mathcal{D}_{e}$ must be stable under the action of $\mathbb{Z}_{2}$, since this action is trivial on $\operatorname{Irr}(\mathcal{D})$. As before, this implies that $\mathcal{C}$ contains a fusion subcategory $\mathcal{B}$ of dimension 144,240 or 360 , respectively, containing the central subcategory $\operatorname{Rep} \mathbb{Z}_{2}$. Hence $\mathcal{B}_{\mathbb{Z}_{2}}=\mathcal{C}(H)$, 
where $H$ is a $\mathbb{Z}_{2}$-stable subgroup of $\mathbb{A}_{6}$ with order 72,120 or 180 , respectively. But $\mathbb{A}_{6}$ has no subgroups neither of order 72,120 nor 180 , therefore the type $(1,24 ; 2,84)$ is also impossible for $\mathcal{D}$.

Suppose finally that $\mathcal{D}$ is a solvable pointed fusion category. We have $\mathcal{D}=$ $\mathcal{C}(\Gamma, \omega)$, where $\omega: \Gamma \times \Gamma \times \Gamma \rightarrow k^{*}$ is a 3-cocyle and $\Gamma$ is a solvable non-abelian subgroup of order 360 . In addition $\mathbb{Z}_{2}$ acts on $\Gamma$ by group automorphisms and the subgroup $\Gamma_{0}$ of fixed points of $\Gamma$ under this $\mathbb{Z}_{2}$-action is of order 24 . Let $S$ be a Sylow 5-subgroup of $\Gamma$. Since $\Gamma$ is solvable, there exist $H$, a Hall $\{2,5\}$ subgroup of $\Gamma$, and $K$, a Hall $\{3,5\}$-subgroup of $\Gamma$, such that $S \subseteq H$ and $S \subseteq K$. A counting argument shows that $S \unlhd H$ and $S \unlhd K$ and so $S \unlhd\langle H, K\rangle=\Gamma$. Hence $S$ is the unique Sylow 5-subgroup of $\Gamma$ and then $S$ is $\mathbb{Z}_{2}$-stable. In this case $\mathcal{C}\left(S,\left.\omega\right|_{S}\right)^{\mathbb{Z}_{2}}$ is a fusion subcategory of $\tilde{\mathcal{C}}$ of dimension 10 , containing the central subcategory $\operatorname{Rep} \mathbb{Z}_{2}$. Therefore $\mathcal{C}$ has a fusion subcategory $\mathcal{B}$ with dimension 10 , containing the central subcategory Rep $\mathbb{Z}_{2}$. The de-equivariantization $\mathcal{B}_{\mathbb{Z}_{2}} \subseteq \mathcal{C}\left(\mathbb{A}_{6}\right)$ is of dimension 5 . Then $\mathcal{B}_{\mathbb{Z}_{2}}=\mathcal{C}(T)$, where $T$ is a $\mathbb{Z}_{2}$-stable subgroup of $\mathbb{A}_{6}$ of order 5 . We have that $T=\{\mathrm{id},($ abcde $),($ acebd $),($ adbec $),($ aedcb $)\}$, and without loss of generality we may assume $a=1$ and $b=2$. We thus reach a contradiction, since $(12)(12 c d e)(12)=(21 c d e) \neq(1 c e 2 d)$. This proves that $\widetilde{\mathcal{C}}$ cannot be solvable and finishes the proof of the theorem.

\section{Solvability AND FUSION RULES OF A BRAIDED FUSION CATEGORY}

Let $\mathcal{C}$ be a fusion category. Suppose that $\mathrm{FPdim} \mathcal{C}$ is an integer (which is always the case if $\mathcal{C}$ is solvable). Then the adjoint subcategory $\mathcal{C}_{a d}$ is integral [11, Proposition 8.27].

Assume that $\mathcal{C}$ is braided. Recall that $\mathcal{C}$ is solvable and integral, then either it is pointed or it contains a nontrivial Tannakian subcategory [29, Proposition 5.2].

Theorem 5.1. Let $\mathcal{C}, \tilde{\mathcal{C}}$ be Grothendieck equivalent braided fusion categories. Suppose that $\mathcal{C}$ is solvable. Then the following hold:

(i) $\tilde{\mathcal{C}}_{p t}$ is a solvable fusion category and it is not trivial if $\tilde{\mathcal{C}}$ is not trivial.

(ii) If $\tilde{\mathcal{C}}$ is not pointed, then it contains a nontrivial Tannakian subcategory.

Proof. Since $\mathcal{C}$ is solvable, [12, Proposition 4.5 (iv)] implies that $\mathcal{C}_{p t} \neq$ Vect. In addition $\mathcal{C}_{p t}$ is a solvable fusion category. Hence $\tilde{\mathcal{C}}_{p t} \neq \operatorname{Vect}$. We have $\mathcal{C}_{p t} \cong$ $\mathcal{C}(G(\mathcal{C}), \omega)$ for some invertible 3-cocycle $\omega$ on $G(\mathcal{C})$. By assumption $\mathcal{C}$ is solvable, hence the group $G(\mathcal{C})$ is solvable.

Moreover, $\tilde{\mathcal{C}}_{p t}$ is Grothendieck equivalent to $\mathcal{C}_{p t}$ and therefore there exists an isomorphism of groups $G(\mathcal{C}) \cong G(\tilde{\mathcal{C}})$. Hence $G(\tilde{\mathcal{C}})$, and a fortiori also $\tilde{\mathcal{C}}_{p t}$, are solvable. This shows part (i).

Suppose that $\tilde{\mathcal{C}}$ is not pointed, so that $\mathcal{C}$ is not pointed neither. Note that $\mathcal{C}_{a d}$ is Grothendieck equivalent to $\tilde{\mathcal{C}}_{a d}$. If $\mathcal{C}_{a d}$ is a proper fusion subcategory, then an inductive argument implies that $\tilde{\mathcal{C}}_{a d}$ is solvable and therefore so is $\tilde{\mathcal{C}}$, because it is a $U(\tilde{\mathcal{C}})$-extension of $\tilde{\mathcal{C}}_{a d}$ and the universal grading group $U(\tilde{\mathcal{C}})$ is abelian. Hence we may assume that $\tilde{\mathcal{C}}_{a d}=\tilde{\mathcal{C}}$ (in particular, the same is true for $\mathcal{C}$ ). Since $\mathcal{C}$ is solvable, then its Frobenius-Perron dimension is an integer and therefore $\mathcal{C}$ is in fact integral. Then $\tilde{\mathcal{C}}$ is also integral. To show part (ii) we may assume that $\tilde{\mathcal{C}}$ is not solvable, in view of [29, Proposition 5.2].

By part (i), $\tilde{\mathcal{C}}_{p t}$ is solvable and not trivial. Note that $\tilde{\mathcal{C}}$ cannot contain any nontrivial non-degenerate fusion subcategory. In fact, if $\mathcal{C}$ were non-degenerate, then $\tilde{\mathcal{C}}_{a d}=\tilde{\mathcal{C}}_{p t}^{\prime} \subsetneq \tilde{\mathcal{C}}$, against the assumption. If, on the other hand, $\tilde{\mathcal{D}} \subseteq \tilde{\mathcal{C}}$ were a proper non-degenerate subcategory, then $\tilde{\mathcal{C}} \cong \tilde{\mathcal{D}} \otimes \tilde{\mathcal{D}}^{\prime}$, and both $\tilde{\mathcal{D}}$ and $\tilde{\mathcal{D}}^{\prime}$ 
are Grothendieck equivalent to fusion subcategories of $\mathcal{C}$. An inductive argument implies that $\tilde{\mathcal{D}}$ and $\tilde{\mathcal{D}}^{\prime}$ are solvable and therefore so is $\tilde{\mathcal{C}}$.

Suppose that $\tilde{\mathcal{C}}$ contains no nontrivial Tannakian subcategory. It follows from 29. Lemma 7.1] that $\tilde{\mathcal{C}}_{p t}=\tilde{\mathcal{C}}^{\prime} \cong$ sVect and $G[\tilde{X}]=\mathbf{1}$, for all simple object $\tilde{X}$ of $\tilde{\mathcal{C}}$. This implies that FPdim $\mathcal{C}_{p t}=2$ and $G[X]=\mathbf{1}$, for all simple object $X$ of $\mathcal{C}$.

On the other hand, since $\mathcal{C}$ is solvable and $\mathcal{C}_{a d}=\mathcal{C}$, then $\mathcal{C}$ must be a $\mathbb{Z}_{p^{-}}$ equivariantization of a fusion category $\mathcal{D}$ for some prime number $p$. In particular $\mathcal{C}$ contains a (pointed) fusion subcategory of dimension 2 , and therefore $p=2$. It follows from Lemma 2.1 that $\mathcal{C}$ has a simple object $X$ of Frobenius-Perron dimension 2. In addition, for every such simple object $X$, we have $G[X]=\mathbf{1}$.

The Nichols-Richmond theorem implies that $\mathcal{C}$ contains a fusion subcategory $\overline{\mathcal{C}}$ of type $(1,2 ; 2,1 ; 3,2)$ or $(1,3 ; 3,1)$ or $(1,1 ; 3,2 ; 4,1 ; 5,1)$; see [30. Theorem 11], [8, Theorem 3.4]. The first possibility cannot hold in this case, because the unique simple object of dimension 2 of $\overline{\mathcal{C}}$ is necessarily stable under the action of $G(\overline{\mathcal{C}}) \cong \mathbb{Z}_{2}$. The second possibility contradicts the assumption that $\mathrm{FPdim} \mathcal{C}_{p t}=2$. The third possibility is also discarded because $\overline{\mathcal{C}}$ must be a solvable fusion category, whence $\overline{\mathcal{C}}_{p t} \neq$ Vect. This contradiction shows that $\tilde{\mathcal{C}}$ must contain a Tannakian subcategory, and hence (ii) holds.

Proposition 5.2. Let $\mathcal{C}$ be a braided fusion category. Suppose that $\mathcal{E} \subseteq \mathcal{C}$ is a Tannakian subcategory. Then $\mathcal{C}$ is solvable if and only if $\mathcal{E}^{\prime}$ is solvable.

Proof. If $\mathcal{C}$ is solvable, then every fusion subcategory of $\mathcal{C}$ is solvable. In particular, $\mathcal{E}^{\prime}$ is solvable, showing the 'only if' direction. Conversely, suppose that $\mathcal{E}^{\prime}$ is solvable. Since $\mathcal{E}$ is a Tannakian subcategory, it is symmetric, and therefore $\mathcal{E} \subseteq \mathcal{E}^{\prime}$. Then $\mathcal{E}$ is solvable. Let $G$ be a finite group such that $\mathcal{E} \cong \operatorname{Rep} G$ as braided fusion categories. Then the group $G$ is solvable, by [12, Proposition 4.5 (ii)].

Consider the $G$-crossed braided fusion category $\mathcal{C}_{G}$, so that $\mathcal{C} \cong\left(\mathcal{C}_{G}\right)^{G}$ is an equivariantization. Furthermore, the category $\mathcal{C}_{G}$ is a $G$-graded fusion category, and the neutral component $\mathcal{C}_{G}^{0}$ of this grading satisfies $\left(\mathcal{C}_{G}^{0}\right)^{G} \cong \mathcal{E}^{\prime}$, , Proposition 4.56 (i)]. Therefore $\mathcal{C}_{G}^{0}$ is solvable. Since $G$ is solvable, then so is $\mathcal{C}_{G}$ and also $\mathcal{C} \cong\left(\mathcal{C}_{G}\right)^{G}$. This proves the 'if' direction and finishes the proof of the proposition.

Remark 5.3. Let $\tilde{\mathcal{C}}$ be a braided fusion category. Suppose that $\tilde{\mathcal{C}}$ is Grothendieck equivalent to a solvable braided fusion category $\mathcal{C}$ and $\tilde{\mathcal{C}}$ is not solvable. Assume in addition that $\mathrm{FP} \operatorname{dim} \tilde{\mathcal{C}}$ is minimum with respect to these properties.

Then $\tilde{\mathcal{C}}$ must satisfy the following conditions:

(i) $\tilde{\mathcal{C}}_{a d}=\tilde{\mathcal{C}}$.

(ii) $\tilde{\mathcal{C}}_{p t} \neq$ Vect is a solvable fusion subcategory and $\left(\tilde{\mathcal{C}}_{p t}\right)^{\prime}=\tilde{\mathcal{C}}$.

(iii) $\tilde{\mathcal{C}}$ contains a nontrivial Tannakian subcategory and for every Tannakian subcategory $\tilde{\mathcal{E}}, \tilde{\mathcal{E}}^{\prime}=\tilde{\mathcal{C}}$.

(iv) $\mathcal{\mathcal { C }}$ contains no proper non-degenerate fusion subcategories.

Indeed, (i) and (iv) can be shown as in the proof Theorem 5.1. (ii) follows from (i) and Lemma 2.5, and (iii) follow from Theorem 5.1 and Proposition 5.2

\section{The Character table of a SPHERICAL Fusion CATEgory}

6.1. Spherical fusion categories. A spherical structure on a fusion category $\mathcal{C}$ is a natural isomorphism of tensor functors $\psi: \operatorname{id}_{\mathcal{C}} \rightarrow()^{* *}$ such that

$$
d_{+}(X)=d_{-}(X)
$$

for all objects $X$ of $\mathcal{C}$, where $d_{ \pm}(X)=\operatorname{Tr}_{ \pm}\left(\mathrm{id}_{X}\right)$, and for every endomorphism $f: X \rightarrow X, \operatorname{Tr}_{ \pm}(f) \in k$ are defined as the compositions

$$
\operatorname{Tr}_{+}(f): \mathbf{1} \longrightarrow X \otimes X^{*} \stackrel{\psi_{X} f \otimes i \mathrm{id}}{\longrightarrow} X^{* *} \otimes X \longrightarrow \mathbf{1}
$$




$$
\operatorname{Tr}_{-}(f): \mathbf{1} \longrightarrow X^{*} \otimes X^{* *} \stackrel{\operatorname{id} \otimes f \psi_{X}^{-1}}{\longrightarrow} X^{*} \otimes X \longrightarrow \mathbf{1}
$$

Let $\mathcal{C}$ be a spherical fusion category, that is, a fusion category endowed with a spherical structure. The quantum dimension of $X \in \mathcal{C}$ is denoted by $d_{X}:=$ $d_{+}(X)=d_{-}(X)$, and the quantum dimension of $\mathcal{C}$ is defined in the form $\operatorname{dim} \mathcal{C}=$ $\sum_{X \in \operatorname{Irr}(\mathcal{C})} d_{X}^{2}$. The quantum trace of an endomorphism $f: X \rightarrow X$ is denoted by $\operatorname{Tr}(f)=\operatorname{Tr}_{+}(f)=\operatorname{Tr}_{-}(f)$. See [9, Subsection 2.4.3], [1], Subsection 2.2].

Recall that a fusion category is called pseudo-unitary if its global dimension coincides with its Frobenius-Perron dimension. By [11, Proposition 8.24], every weakly integral fusion category is pseudo-unitary. It is shown in [11, Proposition 8.23] that every pseudo-unitary fusion category admits a canonical spherical structure with respect to which quantum dimensions of objects coincide with their Frobenius-Perron dimensions.

6.2. Modular categories and $S$-matrices. A premodular category is a braided fusion category equipped with a spherical structure. Equivalently, $\mathcal{C}$ is a braided fusion category endowed with a ribbon structure, that is, a natural automorphism $\theta: \operatorname{id}_{\mathcal{C}} \rightarrow \operatorname{id}_{\mathcal{C}}$ satisfying

$$
\theta_{X \otimes Y}=\left(\theta_{X} \otimes \theta_{Y}\right) c_{Y, X} c_{X, Y}, \quad \theta_{X}^{*}=\theta_{X^{*}},
$$

for all objects $X, Y$ of $\mathcal{C}$ [2, 9, Subsection 2.8.2].

Let $\mathcal{C}$ be a premodular category. The central charge of $\mathcal{C}$ is the ratio

$$
\xi(\mathcal{C})=\frac{\tau^{+}(\mathcal{C})}{\sqrt{\operatorname{dim} \mathcal{C}}},
$$

where $\sqrt{\operatorname{dim\mathcal {C}}}$ is the positive square root and $\tau^{+}(\mathcal{C})=\sum_{X \in \operatorname{Irr}(\mathcal{C})} \theta_{X} d_{X}^{2}$. See [9, Subsection 6.2].

The $S$-matrix of $\mathcal{C}$ is defined in the form $S=\left(S_{X Y}\right)_{X, Y \in \operatorname{Irr}(\mathcal{C})}$, where for all $X, Y \in \operatorname{Irr}(\mathcal{C})$,

$$
S_{X, Y}=\operatorname{Tr}\left(c_{Y, X} c_{X, Y}\right) \in k
$$

is the quantum trace of the squared braiding $c_{Y, X} c_{X, Y}: X \otimes Y \rightarrow Y \otimes X$.

A premodular category $\mathcal{C}$ is called modular if the $S$-matrix is non-degenerate 32 or, equivalently, if it is non-degenerate [9, Proposition 3.7].

If $\mathcal{C}$ is a spherical fusion category, then its Drinfeld center $\mathcal{Z}(\mathcal{C})$ is a modular category of global dimension $\operatorname{dim} \mathcal{Z}(\mathcal{C})=(\operatorname{dim} \mathcal{C})^{2}$ and central charge $\xi(\mathcal{Z}(\mathcal{C}))=1$ [21, [9, Example 6.9].

Suppose that $\mathcal{C}$ is a modular category. Then for every $X, Y, Z \in \operatorname{Irr}(\mathcal{C})$, the multiplicity $N_{X Y}^{Z}$ of $Z$ in the tensor product $X \otimes Y$ is given by the Verlinde formula:

$$
N_{X Y}^{Z}=\frac{1}{\operatorname{dim} \mathcal{C}} \sum_{T \in \operatorname{Irr}(\mathcal{C})} \frac{S_{X T} S_{Y T} S_{Z^{*} T}}{d_{T}},
$$

where $d_{T}$ denotes the quantum dimension of the object $T$ and $\operatorname{dim} \mathcal{C}$ is the quantum dimension of $\mathcal{C}$. See [1, Theorem 3.1.14].

\subsection{S-equivalence of spherical fusion categories.}

Definition 6.1. Let $\mathcal{C}$ and $\mathcal{D}$ be spherical fusion categories. We shall say that $\mathcal{C}$ and $\mathcal{D}$ are $S$-equivalent if there exists a bijection $f: \operatorname{Irr}(\mathcal{Z}(\mathcal{C})) \rightarrow \operatorname{Irr}(\mathcal{Z}(\mathcal{D}))$ such that $f(\mathbf{1})=\mathbf{1}$ and $S_{f(X), f(Y)}=S_{X, Y}$, for all $X, Y \in \operatorname{Irr}(\mathcal{C})$.

The following lemma summarizes some of the main properties of $S$-equivalence.

Lemma 6.2. Let $\mathcal{C}$ and $\mathcal{D}$ be spherical fusion categories and suppose that $f$ : $\operatorname{Irr}(\mathcal{Z}(\mathcal{C})) \rightarrow \operatorname{Irr}(\mathcal{Z}(\mathcal{D}))$ is an $S$-equivalence. Then the following hold: 
(i) $d_{f(X)}=d_{X}$, for all $X \in \operatorname{Irr}(\mathcal{C})$. In particular, $\operatorname{dim} \mathcal{Z}(\mathcal{C})=\operatorname{dim} \mathcal{Z}(\mathcal{D})$.

(ii) $f: \operatorname{Irr}(\mathcal{Z}(\mathcal{C})) \rightarrow \operatorname{Irr}(\mathcal{Z}(\mathcal{D}))$ is a Grothendieck equivalence.

(iii) For every fusion subcategory $\mathcal{E}$ of $\mathcal{Z}(\mathcal{C})$ we have $f\left(\mathcal{E}^{\prime}\right)=f(\mathcal{E})^{\prime}$. In particular, $f(\mathcal{E})$ is symmetric (respectively, non-degenerate) if and only if so is $\mathcal{E}$.

(iv) For every fusion subcategory $\mathcal{E}$ of $\mathcal{Z}(\mathcal{C}), f$ maps the projective centralizer of $\mathcal{E}$ to the projective centralizer of $f(\mathcal{E})$.

Proof. For every simple object $X$ of $\mathcal{Z}(\mathcal{C})$ we have $d_{X}=d_{1} d_{X}=S_{1, X}=S_{1, f(X)}=$ $d_{1} d_{f(X)}=d_{f(X)}$, and we get (i). Now part (ii) follows from (i) and the Verlinde formula (6.2). Part (iii) follows from the fact that two simple objects $X$ and $Y$ centralize each other if and only if $S_{X, Y}=d_{X} d_{Y}$.

We now show part (iv). Let $X$ and $Y$ be simple objects of $\mathcal{C}$. It follows from 9 , Proposition 3.22] that $X$ belongs to the projective centralizer of $Y$ if and only if $X$ belongs to the centralizer of $Y \otimes Y^{*}$. In view of part (iii) this happens if and only if $f(X)$ centralizes $f(Z)$, for all $Z \in \operatorname{Irr}(\mathcal{C})$ such that $N_{Y \otimes Y^{*}}^{Z} \neq 0$. Since, by (ii), $f$ is a Grothendieck equivalence, then $f(Y)^{*}=f\left(Y^{*}\right)$ (Proposition 3.2 (iii)), and it follows that the last condition is equivalent to the condition that $f(X)$ centralizes $f(Y) \otimes f(Y)^{*}$, that is, $f(X)$ belongs to the projective centralizer of $f(Y)$.

Theorem 6.3. Let $\mathcal{C}$ and $\mathcal{D}$ be $S$-equivalent spherical fusion categories. Then $\mathcal{C}$ is group-theoretical if and only if so is $\mathcal{D}$.

Proof. We have that $\mathcal{C}$ is group-theoretical if and only if $\mathcal{Z}(\mathcal{C})$ is group-theoretical. Suppose that this is the case. In particular $\mathcal{Z}(\mathcal{C})$, and therefore also $\mathcal{Z}(\mathcal{D})$, are integral. Since $\mathcal{Z}(\mathcal{C})$ is a modular category, [10, Corollary 4.14] implies that it contains a symmetric subcategory $\mathcal{E}$ such that $\mathcal{E}_{a d}^{\prime} \subseteq \mathcal{E}$. Since every $S$-equivalence preserves centralizers, symmetric subategories and is a Grothendieck equivalence between $\mathcal{Z}(\mathcal{C})$ and $\mathcal{Z}(\mathcal{D})$, this implies that $f(\mathcal{E})$ is a symmetric subcategory of $\mathcal{Z}(\mathcal{D})$ and $f(\mathcal{E})_{a d}^{\prime}=f\left(\mathcal{E}_{a d}^{\prime}\right) \subseteq f(\mathcal{E})$ (see Proposition $3.2($ iv)). Hence $\mathcal{Z}(\mathcal{D})$ and therefore also $\mathcal{D}$ are group-theoretical. This implies the theorem.

Lemma 6.4. Let $G$ and $\Gamma$ be finite groups and let $\omega: G \times G \times G \rightarrow k^{*}$, $\omega^{\prime}$ : $\Gamma \times \Gamma \times \Gamma \rightarrow k^{*}$ be 3 -cocycles on $G$ and $\Gamma$, respectively. Suppose that the categories $\mathcal{C}(G, \omega)$ and $\mathcal{C}\left(\Gamma, \omega^{\prime}\right)$ are $S$-equivalent. Then $G$ is solvable if and only if so is $\Gamma$.

Proof. It is enough to show the 'if' direction. Thus, let us assume that $G$ is solvable. Let $f: \operatorname{Irr}\left(\mathcal{Z}\left(\mathcal{C}\left(\Gamma, \omega^{\prime}\right)\right)\right) \rightarrow \operatorname{Irr}(\mathcal{Z}(\mathcal{C}(G, \omega)))$ be an $S$-equivalence. The center of the category $\mathcal{C}\left(\Gamma, \omega^{\prime}\right)$ contains a Tannakian subcategory $\mathcal{E}$ equivalent to $\operatorname{Rep} \Gamma$ as braided fusion categories. In view of Lemma 6.2 $f(\mathcal{E})$ is a symmetric fusion subcategory of $\mathcal{Z}(\mathcal{C}(G, \omega))$ which is Grothendieck equivalent to Rep $\Gamma$. Being symmetric, $f(\mathcal{E})$ is equivalent as a fusion category to the category $\operatorname{Rep} F$ for some finite group $F$. Then $F$ is solvable because $\mathcal{Z}(\mathcal{C}(G, \omega))$ is solvable, by [12, Proposition 4.5]. Since the categories $\operatorname{Rep} \Gamma$ and $\operatorname{Rep} F$ are Grothendieck equivalent, then the groups $\Gamma$ and $F$ have the same character table. This implies that $\Gamma$ is solvable. Hence $\mathcal{C}\left(\Gamma, \omega^{\prime}\right)$ is solvable, as claimed.

Theorem 6.5. Let $\mathcal{C}$ and $\mathcal{D}$ be $S$-equivalent spherical fusion categories and suppose that $\mathcal{C}$ is group-theoretical. Then $\mathcal{C}$ is solvable if and only if $\mathcal{D}$ is solvable.

Proof. Since $\mathcal{C}$ is group-theoretical, $\mathcal{Z}(\mathcal{C})$ is equivalent to the center of a pointed fusion category $\mathcal{Z}(\mathcal{C}(G, \omega))$, for some finite group $G$ and 3-cocycle $\omega$ on $G$. Hence $\mathcal{Z}(\mathcal{C})$ contains a Tannakian subcategory $\mathcal{E}$ equivalent to $\operatorname{Rep} G$ as braided fusion categories, such that $(\operatorname{dim} \mathcal{E})^{2}=\operatorname{dim} \mathcal{Z}(\mathcal{C})$.

Being Grothendieck equivalent to $\mathcal{Z}(\mathcal{C}), \mathcal{Z}(\mathcal{D})$ is also group-theoretical, in view of Theorem 6.3 Thus $\mathcal{Z}(\mathcal{C})$ is an integral modular category of dimension $(\operatorname{dim} \mathcal{D})^{2}$ 
and central charge 1 . Note in addition that if $f$ is an $S$-equivalence, then $f(\mathcal{E})$ is a symmetric subcategory of $\mathcal{Z}(\mathcal{D})$ such that $\operatorname{dim} \mathcal{Z}(\mathcal{D})=(\operatorname{dim} f(\mathcal{E}))^{2}$. Theorem 4.8 of [10] implies that $\mathcal{Z}(\mathcal{D})$ is equivalent to the center of a pointed fusion category, that is, $\mathcal{Z}(\mathcal{D}) \cong \mathcal{Z}\left(\mathcal{C}\left(\Gamma, \omega^{\prime}\right)\right)$, for some finite group $\Gamma$ and 3-cocycle $\omega^{\prime}$ on $\Gamma$. Then the theorem follows from Lemma 6.4.

\section{REFERENCES}

[1] B. Bakalov, A. Kirillov Jr., Lectures on tensor categories and modular functors, Amer. Math. Soc., 2001.

[2] A. Bruguières, Catégories prémodulaires, modularisations et invariants des variétés de dimension 3, Math. Ann. 316, 215-36 (2000).

[3] A. Bruguières and S. Natale, Exact sequences of tensor categories, Int. Math. Res. Not. 2011 (24), 5644-5705 (2011).

[4] A. Bruguières and S. Natale, Central exact sequences of tensor categories, equivariantization and applications, J. Math. Soc. Japan 66, 257-287 (2014).

[5] S. Burciu and S. Natale, Fusion rules of equivariantizations of fusion categories, J. Math. Phys. 54 DOI: 10.1063/1.4774293 (2013).

[6] P. Deligne, Catégories tensorielles, Mosc. Math. J. 2, 227-248 (2002).

[7] R. Dijkgraaf, V. Pasquier and P. Roche, Quasi-quantum groups related to orbifold models In: Proc. Modern Quantum Field Theory, Tata Institute, Bombay, 375-383, 1990

[8] J. Dong, S. Natale, L. Vendramin, Frobenius property for fusion categories of small integral dimension, J. Algebra Appl. 14, 1550011 (2015).

[9] V. Drinfeld, S. Gelaki, D. Nikshych and V. Ostrik, On braided fusion categories I, Sel. Math. New Ser. 16, 1-119 (2010).

[10] V. Drinfeld, S. Gelaki, D. Nikshych and V. Ostrik, Group-theoretical properties of nilpotent modular categories, preprint arXiv:0704.0195 (2007).

[11] P. Etingof, D. Nikshych and V. Ostrik, On fusion categories, Ann. Math. (2) 162, 581642 (2005)

[12] P. Etingof, D. Nikshych and V. Ostrik, Weakly group-theoretical and solvable fusion categories, Adv. Math. 226, 176-205 (2011).

[13] A. Ganchev, Fusion rings and tensor categories, In: Noncommutative structures in mathematics and physics, Kluwer Academic Publishers, NATO Sci. Ser. II, Math. Phys. Chem. 22, 295-298 (2001).

[14] S. Gelaki and D. Nikshych, Nilpotent fusion categories, Adv. Math. 217, 1053-1071 (2008).

[15] I. M. Isaacs, Character theory of finite groups, Academic Press, 1976.

[16] A. Jedwab and S. Montgomery, Representations of some Hopf algebras associated to the symmetric group $\mathbb{S}_{n}$, Algebr. Represent. Theory 12, 1-17 (2009).

[17] A. Masuoka, Extensions of Hopf algebras, Trab. Mat. 41/99, Fa.M.A.F. (1999)

[18] S. Mattarei, Character tables and metabelian groups, J. London Math. Soc. 46, 92-100, (1992).

[19] S. Mattarei, An example of p-groups with identical character tables and different derived lengths, Arch. Math. 62, 12-20 (1994).

[20] S. Montgomery, S. J. Witherspoon, Irreducible representations of crossed products, J. Pure Appl. Algebra 129, 315-326 (1998).

[21] M. Müger, From subfactors to categories and topology. II. The quantum double of tensor categories and subfactors, J. Pure Appl. Algebra 180, 159-219 (2003).

[22] M. Müger, Galois extensions of braided tensor categories and braided crossed Gcategories, J. Algebra 277, 256-281 (2004).

[23] D. Naidu, D. Nikshych and S. Witherspoon, Fusion subcategories of representation categories of twisted quantum doubles of finite groups, Int. Math. Res. Not. 2009 (22), 4183-4219 (2009).

[24] D. Naidu and E. Rowell, A finiteness property for braided fusion categories, Algebr. Represent. Theory 14, 837-855 (2011).

[25] S. Natale, On group-theoretical Hopf algebras and exact factorizations of finite groups, J. Algebra 270, 199-211 (2003).

[26] S. Natale, Semisolvability of semisimple Hopf algebras of low dimension, Memoirs Amer. Math. Soc. 186 (2007).

[27] S. Natale, Hopf algebra extensions of group algebras and Tambara-Yamagami categories, Algebr. Represent. Theory 13, 673-691 (2010). 
[28] S. Natale, On quasitriangular structures in Hopf algebras arising from exact group factorizations, Commun. Algebra 39, 4763-4775 (2011).

[29] S. Natale, On weakly group-theoretical non-degenerate braided fusion categories, J. Noncommut. Geom. 8, 1043-1060 (2014)

[30] W. D. Nichols and M. B. Richmond, The Grothendieck group of a Hopf algebra, J. Pure Appl. Algebra 106, 297-306 (1996)

[31] J. Siehler, Near-group categories, Alg. Geom. Topol. 3, 719-775 (2003).

[32] V. Turaev, Quantum invariants of knots and 3-manifolds, de Gruyter Studies in Math. 18, Berlin, 1994.

Facultad de Matemática, Astronomía y Física. Universidad Nacional de Córdoba. Ciem - COnicet. Ciudad Universitaria. (5000) Córdoba, Argentina

E-mail address: escanuela@famaf.unc.edu.ar

E-mail address: natale@famaf.unc.edu.ar

URL: http://www.famaf.unc.edu.ar/ natale 